\title{
A Comprehensive Approach to Revealed Preference Theory*
}

\author{
Hiroki Nishimura ${ }^{\dagger} \quad$ Efe A. Ok ${ }^{\ddagger} \quad$ John K.-H. Quah ${ }^{\S}$
}

July 11, 2016

\begin{abstract}
We develop a version of Afriat's Theorem that is applicable to a variety of choice environments, beyond the setting of classical consumer theory. This allows us to devise tests for rationalizability in the context of choice data on lotteries, contingent consumption, and intertemporal consumption. We also establish a version of Richter's Theorem that characterizes the strict rationalizability of choice data with a continuous utility function (rather than simply with a preference that may not be continuous).
\end{abstract}

JEL Classification: D11, D81.

Keywords: revealed preference, rational choice, Afriat's theorem, Richter's theorem, Engel curves, stochastic monotonicity, impatience

\section{INTRODUCTION}

The most basic question in revealed preference analysis is whether the choices made by an agent is consistent with a particular model of decision making. It is usually assumed that there is an observer who records the alternatives chosen by an agent from different feasible sets, which are subsets of a universal set of alternatives. A particular model of decision making will have specific implications on observed choice behavior and ideally one would like to find a condition on the set of observations that is both necessary and sufficient for consistency with that model.

* Earlier versions of this paper were presented at the APET (Seattle), BRIC Conference (St. Andrews), Brown University, CETC (Vancouver), NYU, and European GE Workshop (Paris); we are grateful to the participants at these seminars for their comments.

${ }^{\dagger}$ Department of Economics, UC-Riverside

${ }^{\ddagger}$ Department of Economics and Courant Institute of Applied Mathematics, New York University

$\S$ Department of Economics, Johns Hopkins University and Department of Economics, National University of Singapore 
To state this a bit more precisely, let us denote the universal set of alternatives by $X$ and let $\mathcal{A}$ be a collection of nonempty subsets of $X$; we interpret $\mathcal{A}$ as the collection of feasible sets from which the agent's choices are observed. We denote the agent's choices from a member $A$ of $\mathcal{A}$ by $c(A)$, which is a nonempty subset of $A$. The choice correspondence $c: \mathcal{A} \rightrightarrows X$ encodes the information collected by the observer on the agent's choice behavior, with different models of decision making leading to different properties on $c$. Since it is canonical in economics to assume that one's choices are governed by optimization with respect to a preference relation, it is crucial that we understand what properties on $c$ are consistent with this behavior. This leads to the notion of "rationalization" a preference relation $\succsim$ on $X$ is said to rationalize $c$ if

$$
c(A) \subseteq\{x \in A: x \succsim y \text { for every } y \in A\},
$$

while it is said to strictly rationalize $c$ if

$$
c(A)=\{x \in A: x \succsim y \text { for every } y \in A\} .
$$

A utility function $u: X \rightarrow \mathbb{R}$ is said to rationalize (or strictly rationalize) $c$ if it induces a preference with that property. The more stringent criterion of strict rationalizability is appropriate when there is good reason to believe that unchosen alternatives, i.e., the alternatives in $A \backslash c(A)$, are strictly less preferred to those in $c(A)$.

One of the most influential approaches in revealed preference theory toward identifying rationalizable choice correspondences is due to Afriat (1967). This work is situated in the classical model of consumer demand, where $X$ is taken as $\mathbb{R}_{+}^{n}$ (with $n$ being the number of distinct goods in the market), and $\mathcal{A}$ is a finite collection of linear budget sets. Afriat's Theorem says that so long as the choice correspondence $c$ obeys a fairly intuitive and easy-to-check property called "cyclical consistency," then $c$ can be rationalized by a strictly increasing, continuous, and concave utility function on $\mathbb{R}_{+}^{n}$. The converse of this statement is also true in the sense that any agent who chooses with a locally nonsatiated preference will have a choice correspondence that obeys cyclical consistency.

The significance of Afriat's Theorem is evident from the large body of theoretical and empirical work that it has inspired. However, the application of this result is restricted to the analysis of consumer demand and to those situations where an agent's choice environment could be modeled in a formally similar way. This motivated one of the main objectives of this paper: to develop a version of Afriat's Theorem that is applicable to many other choice environments.

We first show that a generalization of Afriat's cyclical consistency property characterizes those choice correspondences $c$ that are rationalizable by a preference relation on 
$X$ which is (strictly) increasing with respect to a given preorder $\unrhd$ on $X$. This result is obtained without imposing any conditions on $X$ and $\mathcal{A}$. If we further assume that $X$ is a metric space (satisfying some standard regularity conditions) and that $\mathcal{A}$ is a finite collection of feasible sets, then $c$ is rationalizable by a strictly increasing (with respect to $\unrhd)$ and continuous utility function. In particular, if we set $X$ to be $\mathbb{R}_{+}^{n}$ and the preorder $\unrhd$ to be the coordinatewise order on $\mathbb{R}_{+}^{n}$, we recover the fact that (Afriat's) classical cyclical consistency property guarantees the rationalizability of $c$ by a continuous and strictly increasing utility function, which is the essence of Afriat's Theorem. ${ }^{1}$

Of course, the strength of our result is that its applicability goes beyond the classical consumption choice environment. For example, in many experimental studies, observations are collected from a subject who chooses among simple lotteries over monetary outcomes. Assuming that there are $n$ outcomes, the universal set $X$ of alternatives in that setup would be the $(n-1)$-dimensional unit simplex (acting as the set of all possible lotteries), and $\mathcal{A}$ would be a finite collection of subsets of $X$. It is then natural to ask if the subject's choice behavior can be rationalized by a continuous utility function that is increasing with respect to first order stochastic dominance. By choosing $\unrhd$ to be the first order stochastic dominance (partial) order, our theorem provides necessary and sufficient conditions for $c$ to possess this structure. More generally, our theorem can be applied to other contexts where we may wish to determine the rationalizability of a choice correspondence by a utility function satisfying certain properties appropriate to that context. We show how our result can be applied to data collected from an agent choosing contingent consumption, intertemporal consumption, or public policies.

Our extension of Afriat's Theorem has something new to say even in the classical environment of consumer demand. One issue we discuss is recoverability (Varian (1982)), that is, the extent to which an outside observer is able to recover information on the agent's preferences from her observed choices, without subscribing to a particular utility function that happens to rationalize the choice data. We are able to identify the set of all recoverable preference pairs when rationalizing utility functions are allowed to be continuous and strictly increasing. Another issue concerns testing for rationalizability in the case where the observations consist of Engel curves (which are often estimated in empirical studies of consumer demand). Since even a single Engel curve (defined for a given price vector) corresponds to an infinite set of choice observations, Afriat's

\footnotetext{
${ }^{1}$ Our approach does not allow us to recover Afriat's Theorem in its entirety because we cannot guarantee that the rationalizing utility function is concave. Indeed, concavity is not a meaningful property in our setup since we do not assume here that $X$ is a vector space. In addition, our proof is nonconstructive, and thus differs significantly from Afriat's original proof.
} 
Theorem in its original form cannot be applied to test for rationalizability. However, in our approach, we can relax the assumption that $\mathcal{A}$ is finite and we prove that a finite family of Engel curves is rationalizable by a continuous and strictly increasing utility function if, and only if, it satisfies the classical cyclical consistency property.

This paper also contributes to the theory of strictly rationalizable choice correspondences. A very influential paper using this rationalizability criterion is Richter (1966); Richter's Theorem gives necessary and sufficient conditions under which a choice correspondence $c$ is strictly rationalizable, and (unlike Afriat's Theorem) it does so without positing any structure on either $X$ or $\mathcal{A}$. There is, however, a price to pay for this level of generality: with no structure on $X$, it is not even meaningful to specify useful properties such as continuity and/or monotonicity on the rationalizing preference. The second main objective of our paper is to formulate a version of Richter's Theorem in which a rationalizing preference can have these properties. To be precise, when $X$ is a metric space (satisfying some standard regularity conditions), and $\mathcal{A}$ is finite, we show that a generalization of Richter's "congruence axiom" characterizes those choice correspondences that are strictly rationalized by a continuous and strictly increasing (with respect to a given preorder $\unrhd$ ) utility function on $X$. This can be viewed as a continuous and monotone version of Richter's Theorem and, as in our earlier rationalizability results, it is widely applicable.

Last, but not least, we would like to point out a methodological contribution of our paper. Due to differences in their proof methods and in the contexts of their applications, Afriat's Theorem and Ricther's Theorem are often treated separately in the literature. On the other hand, this paper takes a unified approach to both results and our extensions of these theorems allow them to be applicable in exactly the same choice environments. In this way, we are able to clarify the relationship these results and also between the cyclical consistency axiom and the congruence axiom (as well as their generalizations).

The paper is organized as follows. In Section 2 we define the basic concepts used in our analysis and give examples of choice environments where our results are applicable. Our extension of Afriat's Theorem, together with the related extension of Afriat's cyclical consistency property is explained in Section 3. Section 4 is devoted to strict rationalizability and our extension of Richter's Theorem while the applications of our results in a number of specific choice environments are discussed in Section 5. 


\section{CHOICE ENVIRONMENTS}

By a choice environment, we mean an ordered pair $((X, \unrhd), \mathcal{A})$, where $(X, \unrhd)$ is a preordered set and $\mathcal{A}$ is a nonempty collection of nonempty subsets of $X{ }^{2}$ We interpret $X$ as the consumption space, that is, the grand set of all mutually exclusive choice alternatives. We think of $\unrhd$ as an exogenously given dominance relation on $X$, and view the statement $x \unrhd y$ as saying that $x$ is an objectively better alternative than $y$ (in the sense that $x \unrhd y$ implies that every individual in the society prefers $x$ over $y$ ). Finally, $\mathcal{A}$ is interpreted as the set of all feasible sets from which a decision maker is observed to make a choice. For instance, if the data at hand is so limited that we have recorded the choice(s) of an agent in the context of a single feasible set $A \subseteq X$, we would set $\mathcal{A}=\{A\}$. At the other extreme, if we have somehow managed to keep track of the choices of the agent from every possible feasible set $A \subseteq X$ (as is sometimes possible in the controlled environments of laboratory experiments), we would set $\mathcal{A}=2^{X} \backslash\{\varnothing\}$.

By a preference relation on $X$, we mean a complete preorder $\succsim$ on $X$. Such a relation is said to extend $\unrhd$ if (i) $x \succsim y$ whenever $x \unrhd y$, and (ii) $x \succ y$ whenever $x \triangleright y$. (Here $\succ$ and $\triangleright$ stand for the asymmetric parts of $\succsim$ and $\unrhd$, respectively.)

Given a choice environment $((X, \unrhd), \mathcal{A})$, we say that a set-valued map $c: \mathcal{A} \rightrightarrows X$ is a choice correspondence (on $\mathcal{A})$ if $c(A)$ is a nonempty subset of $A$ for each $A \in \mathcal{A}$. A preference relation $\succsim$ on $X$ is said to rationalize the choice correspondence $c$ if

$$
c(A) \subseteq \max (A, \succsim)
$$

for each $A \in \mathcal{A} .^{3}$ We should emphasize that this notion of rationalizability is rather weak; in fact, by itself, it does not impose any structure on choice correspondences since every choice correspondence is rationalizable by the preference relation that declares all alternatives in $X$ indifferent. ${ }^{4}$ The situation becomes more interesting, however, if we also require the preference relation that rationalizes $c$ to extend the dominance relation $\unrhd$, which is a natural requirement since we interpret $\unrhd$ as an "objective" dominance relation that all individuals agree on. We say that a choice correspondence $c$ on $\mathcal{A}$ is

\footnotetext{
${ }^{2}$ Terminology: A binary relation $R$ on $X$ is a nonempty subset of $X \times X$, but as usual, we write $x R y$ instead of $(x, y) \in R$. We say that $R$ is a preorder on $X$ if it is reflexive (that is, $x R x$ for each $x \in X$ ) and transitive (that is, $x R y R z$ implies $x R z$ for each $x, y, z \in X$ ). In turn, we say that $R$ is complete if either $x R y$ or y $R x$ holds for any $x, y \in X$. Finally, the asymmetric part of $R$ is defined as the binary relation $R^{>}$on $X$ such that $x R^{>} y$ iff $x R y$ but not $y R x$. (This relation is never complete, as it is not reflexive.)

${ }^{3}$ Terminology: For any preorder $\succsim$ on a nonempty set $X$, and any nonempty subset $A$ of $X$, we let $\max (A, \succsim)$ stand for the collection of all maximum alternatives in $A$ with respect to $\succsim$, that is, $\max (A, \succsim):=\{x \in A: x \succsim y$ for every $y \in A\}$.

${ }^{4}$ Put formally, $X \times X$ rationalizes any choice correspondence $c: \mathcal{A} \rightrightarrows X$.
} 
$\unrhd$-rationalizable if there is a preference relation $\succsim$ on $X$ that not only rationalizes $c$ but also extends $\unrhd$. Provided that $\unrhd$ is nontrivial in the sense that $x \triangleright y$ holds for some $x, y \in X$, the characterization of all $\unrhd$-rationalizable choice correspondences is a nontrivial problem and this is the central issue addressed in this paper.

The following examples speak to the generality of our framework. To demonstrate the applicability of our main results, we shall return to them later in the paper.

Example 1. (Classical consumption choice environments) Let $n$ be a positive integer, and denote by $\geq$ the coordinatewise ordering on $\mathbb{R}_{+}^{n} \cdot{ }^{5}$ We interpret $n$ to be the number of commodities available for consumption, and the $j$ th entry in $\mathbf{x}=\left(x_{1}, x_{2}, \ldots, x_{n}\right)$ as the agent's level of consumption of good $j$. We denote by $B(\mathbf{p}, I)$ the (linear) budget set at a price vector $\mathbf{p} \in \mathbb{R}_{++}^{n}$ and income $I>0$, that is, $B(\mathbf{p}, I):=\left\{\mathbf{x} \in \mathbb{R}_{+}^{n}: \mathbf{p x} \leq\right.$ $I\}$. A classical consumption choice environment is $\left(\left(\mathbb{R}_{+}^{n}, \geq\right), \mathcal{A}\right)$, where $\mathcal{A}$ is a nonempty subset of $\left\{B(\mathbf{p}, I):(\mathbf{p}, I) \in \mathbb{R}_{++}^{n} \times \mathbb{R}_{++}\right\}$. Afriat's (1967) Theorem, which we discuss in Section 3.1, is situated in this environment and it characterizes the $\geq$ rationalizable choice correspondences $c$ on $\mathcal{A}$ under the assumption that $\mathcal{A}$ is finite. (The $\geq$-rationalization corresponds to rationalization by a preference relation on $\mathbb{R}_{+}^{n}$ where the agent strictly prefers to have more of any good.)

Example 2. (Forges-Minelli choice environments) To deal with nonlinear pricing or quantity restrictions, as well as constraint sets arising in games, some authors have extended Afriat's Theorem by modifying the classical consumption choice environment to allow for nonlinear budget sets (cf. Matzkin (1991) and Chavas and Cox (1993)). In particular, Forges and Minelli (2009) take as a primitive finitely many ordered pairs, say, $\left(A^{1}, \mathbf{x}^{1}\right),\left(A^{2}, \mathbf{x}^{2}\right), \ldots,\left(A^{k}, \mathbf{x}^{k}\right)$, where $A^{i}$ is a subset of $\mathbb{R}_{+}^{n}$ and $\mathbf{x}^{i}$ is contained in $A^{i}$. Each set $A^{i}$ is assumed to be compact and comprehensive; the latter means that if $\mathbf{y} \in A^{i}$, then $\mathbf{z} \in A^{i}$ for any $\mathbf{z} \in \mathbb{R}_{+}^{n}$ such that $\mathbf{z} \leq \mathbf{y}$. They interpret this data as corresponding to the situation in which we observe a decision maker choosing the bundle $\mathbf{x}^{i}$ from the generalized budget set $A^{i}$, for $i=1,2, \ldots, k$. Their setup is captured by the choice environment $\left(\left(\mathbb{R}_{+}^{n}, \geq\right), \mathcal{A}\right)$, where each element of $\mathcal{A}$ is compact and comprehensive. The main result of Forges and Minelli (2009) generalizes Afriat's Theorem by characterizing $\geq$-rationalizable choice correspondences in this environment.

Example 3. (Choice over lotteries) In many experiments designed to check whether subjects are, say, expected utility maximizers, subjects are asked to make choices across a menu of feasible sets that contain simple monetary lotteries. (In particular, the well-

\footnotetext{
${ }^{5}$ Formally, $\mathbf{x} \geq \mathbf{y}$ iff $x_{i} \geq y_{i}$ for each $i=1, \ldots, n$.
} 
known Allais paradox is framed in such a setting.) One could develop conditions in this context that characterize the structure of observations consistent with expected utility maximization (cf. Fishburn (1975)). However, we may wish to take a step back and begin with a more basic question: are the subject's choices rationalizable by some preference relation over lotteries that is consistent with first-order stochastic dominance (even if it may not admit an expected utility representation)? This question can be addressed in our general framework as follows.

Let $\Delta^{n-1}$ stand for the $(n-1)$-dimensional unit simplex in $\mathbb{R}^{n}$, that is, $\Delta^{n-1}:=\{\mathbf{p} \in$ $\left.\mathbb{R}_{+}^{n}: p_{1}+\cdots+p_{n}=1\right\}$. We interpret $\Delta^{n-1}$ as the space of all lotteries whose supports are contained within a given set of $n$ (certain) prizes. Assuming that the prizes are monetary, it makes sense to order the lotteries by the associated first-order stochastic dominance relation, which we shall denote by $\geq_{\mathrm{FSD}} \cdot{ }^{6}$ The choice environment is thus $\left(\left(\Delta^{n-1}, \geq_{\text {FSD }}\right), \mathcal{A}\right)$, where $\mathcal{A}$ is a nonempty collection of nonempty subsets of $\Delta^{n-1}$.

In this paper, we provide a characterization of those choice correspondences on $\mathcal{A}$ that are $\geq_{\text {FSD }}$-rationalizable. Just as the results of Afriat (1967) and Forges and Minelli (2009) provide a practical procedure for testing $\geq$-rationalizability in their respective choice environments, so our characterization provides an empirically implementable test of $\geq_{\text {FSD}^{-}}$rationalizability in the case of choice over lotteries. ${ }^{7}$

\section{RATIONALIZABILITY}

The objective of this section is to extend Afriat's Theorem beyond the confines of the classical consumption choice environment. We begin with an explanation of that classic result.

3.1. Afriat's Theorem. Let $\mathcal{O}$ be a nonempty finite set of ordered pairs $(\mathbf{p}, \mathbf{x})$ in $\mathbb{R}_{++}^{n} \times \mathbb{R}_{+}^{n} \backslash\{\mathbf{0}\}$. Each $(\mathbf{p}, \mathbf{x}) \in \mathcal{O}$ is interpreted as the observation that the consumer has chosen the consumption bundle $\mathbf{x}$ at the price vector $\mathbf{p}$. (Notice that all prices are assumed to be strictly positive, but the consumer's purchases of some (but not all) goods may be zero.) The main problem addressed in Afriat (1967) is to determine the conditions under which the set of observations $\mathcal{O}$ is consistent with the maximization of

\footnotetext{
${ }^{6}$ Assuming that prize 1 is the lowest prize, prize 2 is the second lowest, and so on, we have $\mathbf{p} \geq_{\text {FSD }} \mathbf{q}$ iff $p_{1}+\cdots+p_{j} \leq q_{1}+\cdots+q_{j}$ for each $j=1, \ldots, n$.

${ }^{7} \mathrm{It}$ is also interesting to ask rationalizability-type questions in the case where observations consist of choices made over acts (which are maps from states to outcomes); two examples are Green and Osband (1991) and Bossert and Suzumura (2010). The former studies consistency with expected utility maximization in the case where state probabilities are known. The latter addresses a similar issue but instead imposes a weak restriction on preferences (over acts) that does not appeal to state probabilities. A third example is our study of the demand for contingent consumption in Section 5.4.
} 
a strictly increasing utility function. In other words, it asks which data sets $\mathcal{O}$ would admit a strictly increasing function $u: \mathbb{R}_{+}^{n} \rightarrow \mathbb{R}$ such that

$$
u(\mathbf{x}) \geq u(\mathbf{y}) \text { for all } \mathbf{y} \in B(\mathbf{p}, \mathbf{p x})
$$

for each $(\mathbf{p}, \mathbf{x}) \in \mathcal{O}$. Note that there is no loss of generality in assuming that $\mathbf{p x}=1$ for each $(\mathbf{p}, \mathbf{x}) \in \mathcal{O} .^{8}$ and we shall adopt this normalization in what follows.

We say that $\mathcal{O}$ satisfies the cyclical consistency condition if

$$
\mathbf{p}^{2} \mathbf{x}^{1} \leq \mathbf{p}^{2} \mathbf{x}^{2}, \ldots, \mathbf{p}^{k} \mathbf{x}^{k-1} \leq \mathbf{p}^{k} \mathbf{x}^{k} \text { and } \mathbf{p}^{1} \mathbf{x}^{k} \leq \mathbf{p}^{1} \mathbf{x}^{1}
$$

implies that every one of these inequalities holds as an equality, for any integer $k \geq 2$ and $\left(\mathbf{p}^{1}, \mathbf{x}^{1}\right), \ldots,\left(\mathbf{p}^{k}, \mathbf{x}^{k}\right) \in \mathcal{O}$. (This property is also commonly known in its equivalent formulation, due to Varian (1982), as the generalized axiom of revealed preference.) One may readily check that if $\mathcal{O}$ is consistent with utility maximization with respect to a strictly increasing utility function, then it must satisfy the cyclical consistency condition. ${ }^{9}$ The nontrivial part of Afriat's Theorem says that cyclical consistency is in fact also sufficient for $\mathcal{O}$ to be consistent with the maximization of a continuous, concave and strictly increasing utility function. ${ }^{10}$

To state Afriat's Theorem in the language of choice environments, let $P$ be the set of price vectors observed in $\mathcal{O}$, that is, $P:=\{\mathbf{p}:(\mathbf{p}, \mathbf{x}) \in \mathcal{O}\}$, and put $\mathcal{A}:=\{B(\mathbf{p}, 1)$ : $\mathbf{p} \in P\}$. The choice environment $\left(\left(\mathbb{R}_{+}^{n}, \geq\right), \mathcal{A}\right)$ is a special case of the environment we introduced in Example 1. Next, we define the choice correspondence $c$ on $\mathcal{A}$ by $c(B(\mathbf{p}, 1)):=\{\mathbf{x}:(\mathbf{p}, \mathbf{x}) \in \mathcal{O}\}$. Since there is a one-to-one correspondence between the sets $\mathcal{A}$ and $P$, we can identify $c$ with the (normalized) demand correspondence $d$ :

\footnotetext{
${ }^{8}$ Given $\mathcal{O}$, put $\mathcal{O}^{\prime}:=\left\{\left(\frac{1}{\mathbf{p x}} \mathbf{p}, \mathbf{x}\right):(\mathbf{p}, \mathbf{x}) \in \mathcal{O}\right\}$, and notice that $\mathcal{O}$ is consistent with utility maximization iff $\mathcal{O}^{\prime}$ is consistent with utility maximization, while $\mathbf{q x}=1$ for every $(\mathbf{q}, \mathbf{x}) \in \mathcal{O}^{\prime}$.

${ }^{9}$ In fact, $\mathcal{O}$ obeys cyclical consistency so long as it is collected from an agent with a locally nonsatiated preference.

10 To elaborate, the usual proof of this result (see, for example, Varian (1982)) shows that cyclical consistency implies that a certain set of linear inequalities (usually referred to as the Afriat inequalities) admits a solution. With this solution, one could explicitly construct a utility function $u$ on $\mathbb{R}_{+}^{n}$ that rationalizes the data. In the case when prices are strictly positive, this utility function is continuous, concave, and strictly increasing. In fact, Afriat's Theorem also allows for observed prices in the data set to vanish for some goods; when that happens, the rationalizing utility function is still continuous and concave, but it is no longer strictly increasing. Instead, $u$ has the following weaker property: $u(y)>u(x)$ whenever $y \gg x$ (that is, whenever $y_{i}>x_{i}$ for every good $i$ ). In this paper, we focus on Afriat's Theorem in the case when the prices observed in the data set are all strictly positive, which is empirically more relevant. This case is convenient because we can then set the dominance relation as $\geq$, which is a continuous preorder, whereas the binary relation $\gg$ is less well-behaved. Indeed, the topological properties of the dominance relation will be relevant when we consider continuous rationalizability (see Section 3.4).
} 
$P \rightrightarrows \mathbb{R}_{+}^{n}$ (associated with $\mathcal{O}$ ) defined by $d(p):=\{\mathbf{x}:(\mathbf{p}, \mathbf{x}) \in \mathcal{O}\}$. At the cost of a slight abuse of terminology, we say that $d$ is $\geq$-rationalizable if $c$ is $\geq$-rationalizable, and that $d$ (or $c$ ) is cyclically consistent if (2) implies that every one of those inequalities holds as an equality, for any integer $k \geq 2, \mathbf{p}^{1}, \ldots, \mathbf{p}^{k} \in P$ and $\mathbf{x}^{1} \in d\left(\mathbf{p}^{1}\right), \ldots, \mathbf{x}^{k} \in$ $d\left(\mathbf{p}^{k}\right)$. Afriat's Theorem says, in particular, that $d$ is $\geq$-rationalizable iff it is cyclically consistent. Moreover, according to this theorem, cyclical consistency of $d$ ensures its rationalizability by a preference relation on $\mathbb{R}_{+}^{n}$ that admits a continuous, concave and strictly increasing utility representation.

3.2. Cyclical consistency in general choice environments. In order to formulate a version of Afriat's Theorem applicable in any choice environment, we must first generalize the cyclical consistency property for choice correspondences that are defined in an arbitrary choice environment. We introduce such a generalization next.

Let $((X, \unrhd), \mathcal{A})$ be a choice environment. For any member $A$ of $\mathcal{A}$, we write $A^{\downarrow}$ to denote the decreasing closure of $A$ with respect to the preorder $\unrhd$ on $X$, that is,

$$
A^{\downarrow}:=\{x \in X: y \unrhd x \text { for some } y \in A\},
$$

and let $A \downarrow$ stand for the strictly decreasing closure of $A$ with respect to $\unrhd$, that is,

$$
A^{\downarrow \downarrow}:=\{x \in X: y \triangleright x \text { for some } y \in A\} .
$$

We say that a choice correspondence $c$ on $\mathcal{A}$ satisfies cyclical $\unrhd$-consistency if, for every integer $k \geq 2$ and $A_{1}, \ldots, A_{k} \in \mathcal{A}$,

$$
x_{1} \in c\left(A_{1}\right) \cap A_{2}^{\downarrow}, \ldots, x_{k-1} \in c\left(A_{k-1}\right) \cap A_{k}^{\downarrow} \text { and } x_{k} \in c\left(A_{k}\right) \cap A_{1}^{\downarrow}
$$

imply

$$
x_{1} \in A_{2}^{\downarrow} \backslash A_{2}^{\downarrow \downarrow}, \ldots, x_{k-1} \in A_{k}^{\downarrow} \backslash A_{k}^{\downarrow \downarrow} \text { and } x_{k} \in A_{1}^{\downarrow} \backslash A_{1}^{\downarrow \downarrow} .
$$

If we choose $k=2$ with $A=A_{1}=A_{2}$ and $x_{1}=x_{2} \in c(A)$, then cyclical $\unrhd$-consistency implies $c(A) \subseteq A \backslash A^{\downarrow \downarrow}$ for each $A \in \mathcal{A}$; in other words, for every feasible set $A$, the agent's choices are not dominated (in terms of $\unrhd$ ) within $A .{ }^{11}$

It is not hard to check that, in a classical consumption choice environment, cyclical $\unrhd$ consistency is equivalent to Afriat's original cyclical consistency axiom and so we could think of cyclical $\unrhd$-consistency as a generalization of Afriat's concept to an arbitrary choice environment. We shall see that this more general concept plays a role in an

\footnotetext{
${ }^{11}$ In the case where $\unrhd$ is a partial order (that is, $x \unrhd y \unrhd x$ holds if, and only if, $x=y$ ), then (4) could be re-stated as requiring $x_{1} \in A_{2} \backslash A_{2}^{\downarrow \downarrow}, \ldots, x_{k-1} \in A_{k} \backslash A_{k}^{\downarrow \downarrow}$ and $x_{k} \in A_{1} \backslash A_{1}^{\downarrow \downarrow}$.
} 
arbitrary choice environment that is analogous to that played by cyclical consistency in the classical environment.

It will be quite important for us to express cyclical $\unrhd$-consistency of $c$ by using the revealed preference relations induced by $c$, and there is indeed a succinct way of doing this. Let us first recall that the transitive closure of a reflexive binary relation $R$ on $X$, which is denoted by $\operatorname{tran}(R)$, is the smallest preorder on $X$ which contains $R .^{12}$ We define the binary relation $R(c)$ on $X$ by

$$
x R(c) y \quad \text { if and only if } \quad(x, y) \in c(A) \times A \text { for some } A \in \mathcal{A} \text {. }
$$

This relation, introduced first by Samuelson (1938) in the special case of consumption problems, is often called the direct revealed preference relation induced by $c$, while the transitive closure of $R(c)$ is referred to as the revealed preference relation induced by $c$. The motivation for this terminology is clear: if $c$ is rationalizable by some preference relation $\succsim$ on $X$, then $x \operatorname{tran}(R(c)) y$ implies $x \succsim y$.

Now, there is no reason for the union of $R(c)$ and $\unrhd$ to be transitive, but the transitive closure of $R(c) \cup \unrhd$ is a preorder on $X$. This preorder is related to $\unrhd$-rationalizability in essentially the same way that the revealed preference relation is linked to rationalizability. Indeed, if $c$ is $\unrhd$-rationalizable by some preference relation $\succsim$ on $X$, then $x \operatorname{tran}(R(c) \cup \unrhd) y$ implies $x \succsim y$. The next result uses this preorder to provide a characterization of cyclical $\unrhd$-consistency that is useful in helping us develop a generalized version of Afria't Theorem.

Proposition $1 .{ }^{13}$ Let $((X, \unrhd), \mathcal{A})$ be a choice environment. Then, a choice correspondence $c$ on $\mathcal{A}$ satisfies cyclical $\unrhd$-consistency if, and only if,

$$
x \operatorname{tran}(R(c) \cup \unrhd) y \quad \text { implies } \quad \text { not } y \triangleright x
$$

for any $x, y \in X$.

3.3. Generalizing Afriat's Theorem. It is quite clear that for a choice correspondence $c$ to be $\unrhd$-rationalizable by some preference relation $\succsim$, it is necessary that it obeys cyclical $\unrhd$-consistency. Indeed, consider any $A_{1}, \ldots, A_{k} \in \mathcal{A}$ such that (3) holds. Since $x_{1} \in A_{2}^{\downarrow}$ and $x_{2} \in c\left(A_{2}\right)$, we know that $x_{2} \succsim x_{1}$. Repeating this observation, we obtain $x_{k} \succsim x_{k-1} \succsim \ldots x_{1}$. Given that $x_{k} \in A_{1}^{\downarrow}$, there is a $y \in A_{1}$ such that $y \unrhd x_{k}$. On the other

\footnotetext{
${ }^{12}$ It is easily verified that $x \operatorname{tran}(R) y$ iff there exist a positive integer $k$ and $x_{0}, \ldots, x_{k} \in X$ such that $x=x_{0} R x_{1} R \cdots R x_{k}=y$.

13 This fact was kindly suggested to us by an anonymous referee of this journal; we are grateful to him/her.
} 
hand $x_{k} \succsim x_{1} \succsim y$ since $x_{1} \in c\left(A_{1}\right)$, which therefore excludes the possibility that $y \triangleright x_{k}$. We conclude that $x_{k} \in A_{1}^{\downarrow} \backslash A_{1}^{\downarrow \downarrow}$. In a similar way, we can show that $x_{i} \in A_{i+1}^{\downarrow} \backslash A_{i+1}^{\downarrow \downarrow}$ for $i=1,2, \ldots, k-1$.

The following result establishes that cyclical $\unrhd$-consistency is not just necessary but also sufficient for $\unrhd$-rationalizability.

Theorem 2. Let $((X, \unrhd), \mathcal{A})$ be a choice environment. Then, a choice correspondence c on $\mathcal{A}$ satisfies cyclical $\unrhd$-consistency if, and only if, it is $\unrhd$-rationalizable.

Theorem 2 extends the coverage of Afriat's Theorem to the context of an arbitrary choice environment. While it does capture the choice-theoretic gist of Afriat's Theorem, this result does not recover Afrait's result exactly, even when we specialize to the classical consumption choice environment. The rationalizing preference relation in Afriat's Theorem is representable by a continuous, concave and strictly increasing utility function on $\mathbb{R}_{+}^{n}$, while Theorem 2 only guarantees rationalization by a preference relation that extends the coordinate-wise order $\geq$. However, notice that Afriat's Theorem assumes that the number of observations is finite, and this is crucial to the stronger conclusions obtained. Reny (2015) has provided an extension of Afriat's Theorem (in the classical consumption choice environment) where finiteness of the data set is not assumed; the rationalizing preference relation he obtains is representable by a utility function that is increasing and quasiconcave but Reny shows also that it is in general not possible to guarantee the continuity and/or concavity of this function.

Our proof of Theorem 2 is also quite different from the one used by Afriat for his result. Afriat's proof relies very much on the linear structure of $\mathbb{R}^{n}$, which makes it inapplicable in our general context. Instead, our proof uses Szpilrajn's Theorem to guarantee the existence of a complete extension of the preorder $\operatorname{tran}(R(c) \cup \unrhd) \cdot{ }^{14} \mathrm{We}$ then check that this complete preorder rationalizes $c$, and use Proposition 1 to show that it also extends $\unrhd$. While our method of proof is nonconstructive, it has the advantage of yielding a characterization of rationalizability in the context of any choice environment. Moreover, this method, being purely order-theoretic, highlights the connection between the fundamental rationalizability results of Afriat (1967) and Richter (1966). (We discuss this issue in greater detail in Section 4.)

3.4. Continuous rationalizability in general choice environments. In empirical studies using revealed preference methods, we would often wish to go beyond "explaining" the observed choice data to making out-of-sample predictions. Formulating this

\footnotetext{
${ }^{14}$ Szpilrajn's Theorem says that every preorder admits a complete extension. It is well-known in set theory that this theorem cannot be proved without the use of at least some form of the axiom of choice.
} 
within our framework, given a choice environment $((X, \unrhd), \mathcal{A})$, the observer may well be interested in the agent's choice behavior, not only over the members of $\mathcal{A}$, but also across a collection $\mathcal{B}$ of choice problems which is potentially a much larger (and possibly infinite) subset of $2^{X}$. In that case, $\mathcal{A}$ is interpreted as a (random) sample of feasible sets drawn from $\mathcal{B}$, and the observer's hypothesis is that the agent chooses according to some preference relation on $X$ (with given characteristics) for every set in $\mathcal{B}$.

If a given choice correspondence $c$ on $\mathcal{A}$ is rationalizable, the rationalizing preference relation, say, $\succsim$, could be used to make (set-valued) predictions of the agent's behavior across $\mathcal{B}$. However, this research program hinges on the existence of an optimum with respect to $\succsim$ in any member of $\mathcal{B}$, which is generally not true (at least when some of the members of $\mathcal{B}$ are infinite). This issue would be resolved if we endow $X$ with a topology in which each element of $\mathcal{B}$ is compact, and there is a rationalizing preference relation $\succsim$ on $X$ that is upper semicontinuous. Beyond this, it is often also convenient for the set of optimal choices (with respect to $\succsim$ ) to vary continuously with the sets in $\mathcal{B}$ and that would typically require that the preference relation be lower semi-continuous as well. ${ }^{15}$ For these reasons, it is important to find conditions under which a choice correspondence can be rationalized by a continuous preference relation. Note also that whenever $X$ is the Euclidean space (or, more generally, a separable metric space), any continuous preference relation on $X$ will have a continuous utility representation.

This prompts the following definitions. Let $((X, \unrhd), \mathcal{A})$ be a choice environment where $X$ is a topological space. A function $u: X \rightarrow \mathbb{R}$ is strictly $\unrhd$-increasing if (i) $u(x) \geq u(y)$ whenever $x \unrhd y$, and (ii) $u(x)>u(y)$ whenever $x \triangleright y$. (If $X$ is a subset of $\mathbb{R}^{n}$ and $\geq$ is the coordinatewise order, then to say that $u$ is strictly $\geq$-increasing is the same as saying it is strictly increasing in the usual sense.) We say that a choice correspondence $c$ on $\mathcal{A}$ is $\unrhd$-rationalizable by a continuous utility function if there is a continuous and strictly $\unrhd$-increasing function $u: X \rightarrow \mathbb{R}$ such that

$$
c(A) \subseteq \arg \max \{u(x): x \in A\}
$$

for every $A \in \mathcal{A}$.

As we have noted in Section 3.1, Afriat's Theorem guarantees that the rationalizing utility function is continuous (amongst other properties). This conclusion relies on the particular structure of the classical consumption choice environment, $\left(\left(\mathbb{R}_{+}^{n}, \geq\right), \mathcal{A}\right)$. First,

\footnotetext{
${ }^{15}$ For example, a demand correspondence is upper hemicontinuous with respect to prices if the consumer is maximizing a continuous preference; upper semi-continuity of the preference would not suffice for the upper hemi-continuity of demand. More generally, if we endow $\mathcal{B}$ with the Hausdorff metric, then the correspondence mapping $B$ to $\max (B, \succsim)$ is upper hemicontinuous on $\mathcal{B}$ if $\succsim$ is continuous.
} 
$\mathbb{R}_{+}^{n}$ possesses a well-behaved topological structure, so it is meaningful to talk about the continuity of preference relations defined on it. Second, Afriat's Theorem assumes that the observer makes finitely many observations, which means (in our terminology) that $c$ is a finite-valued map on a finite domain $\mathcal{A} .^{16}$

It turns out that analogous results can be established in other choice environments, thereby yielding a generalization of Afriat's Theorem, provided that they are endowed with a well-behaved topological structure. In particular, let us consider $((X, \unrhd), \mathcal{A})$ where (i) $X$ is a locally compact and separable metric space, (ii) $\unrhd$ is a continuous preorder, ${ }^{17}$ and (iii) $\mathcal{A}$ is a collection of nonempty compact subsets of $X$. In what follows, we refer to any such choice environment as a continuous choice environment. Our "rationalization by a utility function" type results will all be proved within the context of such environments. The following is a major case in point.

Theorem 3. Let $((X, \unrhd), \mathcal{A})$ be a continuous choice environment such that $\mathcal{A}$ is a finite set. Then, a closed-valued choice correspondence $c$ on $\mathcal{A}$ satisfies cyclical $\unrhd$-consistency if, and only if, it is $\unrhd$-rationalizable by a continuous utility function.

It is possible to replace the finiteness requirement on $\mathcal{A}$ here with a continuity property. In particular, the following result shows that if, in the context of Theorem $3, \mathcal{A}$ is not finite, but the preorder $\operatorname{tran}(R(c) \cup \unrhd)$ on $X$ is known to be continuous, then the conclusion of that theorem remains valid. As we shall demonstrate later, in certain applications in which $\mathcal{A}$ is not finite, this requirement may be checked directly.

Theorem 4. Let $((X, \unrhd), \mathcal{A})$ be a continuous choice environment, and $c$ a choice correspondence on $\mathcal{A}$ such that $\operatorname{tran}(R(c) \cup \unrhd)$ is a continuous preorder on $X$. Then, c satisfies cyclical $\unrhd$-consistency if, and only if, it is $\unrhd$-rationalizable by a continuous utility function.

Similar to our proof of Theorem 2, and unlike the standard proofs of Afriat's Theorem, Theorems 3 and 4 are obtained by means of a nonconstructive method. The proofs are based on a suitable extension of Szpilrajn's Theorem that allows one to "continuously complete" a continuous preorder. This result is known in topological order theory

\footnotetext{
${ }^{16}$ In the classical consumption choice environment, Reny (2015) provides sufficient conditions under which a (not necessarily finite) data set can be rationalized by an upper semicontinuous utility function. See also Mas-Colell (1978), which studies the rationalizability of demand functions with continuous and upper semicontinuous utility functions; in this case it is assumed that the demand function is specified at all strictly positive prices and incomes (in other words, using our notation, $\mathcal{A}$ consists of all compact linear budget sets).

17 Terminology: We say that a binary relation on a topological space $X$ is continuous if it is a closed subset of the product space $X \times X$.
} 
as Levin's Theorem, and it is stated and proved in the Appendix.

When we apply Theorem 3 to the classical consumption choice environment, assuming that $\mathcal{A}$ is finite and $c$ is closed-valued, we obtain the conclusion that $c$ obeys cyclical consistency iff it is rationalizable by a strictly increasing and continuous utility function. So we effectively recover Afriat's Theorem, though there are two notable differences in the results. First, while Afriat's Theorem assumes that $c$ is finite-valued, our result only requires that $c$ be closed-valued. The second difference is that we do not guarantee that the rationalizing utility function is concave; in turns out that there is a noteworthy reason for this difference, which we shall discuss in greater detail Section 3.6.

We now apply Theorem 3 to the other two examples considered in Section 2.

Example 2 (continued). The Forges-Minelli choice environment $\left(\left(\mathbb{R}_{+}^{n}, \geq\right), \mathcal{A}\right)$ is a continuous choice environment since the elements of $\mathcal{A}$ are assumed to be compact. They are also comprehensive, which means that $A^{\downarrow}=A$ for each $A$ in $\mathcal{A}$. Consequently, the cyclical $\geq$-consistency of a choice correspondence $c$ can be more simply stated in the following way: for every integer $k \geq 2$ and $A_{1}, \ldots, A_{k} \in \mathcal{A}$, if $\mathbf{x}_{1} \in c\left(A_{1}\right) \cap A_{2}, \ldots, \mathbf{x}_{k-1} \in$ $c\left(A_{k-1}\right) \cap A_{k}$ and $\mathbf{x}_{k} \in c\left(A_{k}\right) \cap A_{1}$, then $\mathbf{x}_{1} \in A_{2} \backslash A_{2}^{\downarrow \downarrow}, \ldots, \mathbf{x}_{k-1} \in A_{k} \backslash A_{k}^{\downarrow \downarrow}$ and $\mathbf{x}_{k} \in A_{1} \backslash A_{1}^{\downarrow \downarrow}$. Theorem 3 says that so long as $\mathcal{A}$ is finite and $c$ is closed-valued, then $c$ is rationalizable by a strictly increasing and continuous utility function iff it obeys cyclical $\geq$-consistency. This recovers the result of Forges and Minelli (2009) and, in fact, generalizes it somewhat since we assume here only that $c$ is closed-valued (instead of finite-valued).

Example 3 (continued). In this case the choice environment is $\left(\left(\Delta^{n-1}, \geq_{\mathrm{FSD}}\right), \mathcal{A}\right)$, where the elements of $\Delta^{n-1}$ are interpreted as simple lotteries with monetary outcomes, and the lotteries are partially ordered by the first order stochastic dominance relation $\geq_{\text {FSD }}$. If we endow $\Delta^{n-1}$ with the Euclidean metric and assume that the elements of $\mathcal{A}$ are compact sets in $\Delta^{n-1}$, then this choice environment is continuous. Furthermore, let us assume that the collection $\mathcal{A}$ is finite and we have observed a closed-valued choice correspondence $c$ on $\mathcal{A}$. (In experimental settings, it is not uncommon for $\mathcal{A}$ to be a finite collection of finite sets, in which case these conditions are satisfied immediately.) Theorem 3 tells us that there is a continuous and $\geq_{\text {FSD }}$-increasing utility function on $\Delta^{n-1}$ that rationalizes $c$ iff $c$ satisfies cyclical $\geq{ }_{\text {FSD }}$-consistency. ${ }^{18}$

3.5. Empirical tests of rationalizability. Theorem 3 may be utilized in empirical

\footnotetext{
18 There are other dominance relations that one could reasonably impose in the context of choice over lotteries. An alternative that is more stringent than first order stochastic dominance is second order stochastic dominance, which also induces a continuous partial order $\geq_{\mathrm{SSD}}$ on $\Delta^{n-1}$; in this case, rationalizability would involve checking for cyclical $\geq_{\mathrm{SSD}}$-consistency.
} 
applications in an arbitrary choice environment $((X, \unrhd), \mathcal{A})$, in much the same way that Afriat's Theorem is used in studying consumption choice. Indeed, empirical revealed preference studies are usually based on finite data sets, which ensures that $\mathcal{A}$ is finite and the observed choice correspondence $c$ is finite-valued. In view of Theorem 3, the issue reduces to checking whether or not $c$ satisfies cyclical $\unrhd$-consistency. In general, one may appproach this problem using the following four-step procedure.

(Step I) Check that $c(A) \subseteq A \backslash A^{\downarrow \downarrow}$ for each $A \in \mathcal{A}$.

(Step II) Let $c(\mathcal{A})$ stand for the set of all choices across all choice problems, that is, $c(\mathcal{A}):=$ $\bigcup\{c(A): A \in \mathcal{A}\}$. Construct the binary relation $\mathcal{S}$ on $c(\mathcal{A})$ in the following manner: $x \mathcal{S} y$ if there is an $A \in \mathcal{A}$ such that $x \in c(A)$ and $y \in A^{\downarrow} .{ }^{19}$

(Step III) Identify all cycles in $\mathcal{S}$, say, by using Warshall's algorithm (see Varian (1982)).

(Step IV) Check whether (4) holds for every cycle found in Step III.

This description sets out a procedure for checking cyclical $\unrhd$-consistency that is analogous to the familiar one used to check for cyclical consistency. It is well known that verifying cyclical consistency in practice is computationally undemanding. Whether this is also true of cyclical $\unrhd$-consistency property depends on the complexity of $\unrhd$ and the structure of the sets in $\mathcal{A}$, but it seems likely that it will also be relatively straightforward in the context of many empirical applications. ${ }^{20}$

3.6. A comparison with Afriat's Theorem. At this point it is worth returning again to the Afriat's consumption choice model in order to highlight the distinction between the rationalization obtained here and those obtained in Afriat's analysis and Reny's (2015) extension of that analysis to the case of infinite choice data. Both of those analyses yield a rationalizing preference relation that can be represented by a quasiconcave utility function on $\mathbb{R}_{+}^{n}$. On the other hand, neither Theorem 2 nor 3 says anything about the convexity of the rationalizing preference relation. There is in fact a noteworthy reason for this. The rationalizing preference relation constructed through our approach has actually another property which, in certain cases, precludes the property of convexity. The result below gives more information about the structure

\footnotetext{
${ }^{19}$ If $c(\mathcal{A})$ has $m$ elements, then there are at most $m(m-1)$ nontrivial checks that one has to perform in the construction of $\mathcal{S}$. (Note that $x_{1}, \ldots, x_{k} \in X$ obey (3) iff they form a cycle in $\mathcal{S}$ in the sense that $x_{1} \mathcal{S} x_{k} \mathcal{S} x_{k-1}, \ldots x_{2} \mathcal{S} x_{1}$.)

${ }^{20}$ For an empirical implementation of this testing procedure in the case where $\unrhd$ ranks contingent consumption bundles based on first order stochastic dominance, see Polisson et al. (2013) and the related discussion in Section 5.4.
} 
of this preference relation, and highlights a fundamental difference between Afriat-type constructions and ours.

Proposition 5. Let $((X, \unrhd), \mathcal{A})$ be a choice environment and $c$ a choice correspondence on $\mathcal{A}$ that satisfies cyclical $\unrhd$-consistency. Then, there is a preference relation $\succsim$ on $X$ that (i) extends $\unrhd$, (ii) rationalizes $c$, and (iii) satisfies

$$
x \in \max (A, \succsim) \quad \text { if, and only if, } \quad x \operatorname{tran}(R(c) \cup \unrhd) y \text { for some } y \in c(A)
$$

for every $A \in \mathcal{A}$ and $x \in A$. Moreover, under the conditions of Theorem 4, we can choose $\succsim$ to have a continuous utility representation.

The "if" part of (7) is clear: indeed, for any preference relation $\succsim$ that extends $\unrhd$ and rationalizes $c$, it must be the case that if $x \in A$ and $x \operatorname{tran}(R(c) \cup \unrhd) y$ for some $y \in c(A)$, then $x \in \max (A, \succsim)$. The non-trivial conclusion of this proposition is that, under the hypothesis of cyclical $\unrhd$-consistency, we can find a preference relation that not only extends $\unrhd$ and rationalizes $c$, but for which the "only if" part of (7) also holds. In other words, on any feasible set $A \in \mathcal{A}$, such a preference relation will have more optimal points besides the ones in $c(A)$ only if it is 'required' to do so by the correspondence $c$ itself.

To wit, suppose we have two observations of a consumer in a classical consumption choice environment. In both observations, the prices are $\mathbf{p}$, with the consumer buying a bundle $\mathbf{x}^{1}$ at one observation and $\mathbf{x}^{2}$ at another, with $\mathbf{p} \mathbf{x}^{1}=\mathbf{p} \mathbf{x}^{2}=1$ and $\mathbf{x}^{1} \neq \mathbf{x}^{2}$. Rationalizing these demands by a convex preference relation would entail that every bundle on the line segment between $\mathbf{x}^{1}$ and $\mathbf{x}^{2}$ is also optimal for the individual at prices p. By contrast, Proposition 5 says that there is a preference relation that declares only $\mathbf{x}^{1}$ and $\mathbf{x}^{2}$ as optimal at prices $\mathbf{p}$. Such a preference relation is, per force, not convex.

\section{STRICT RATIONALIZABILITY}

4.1. Richter's Theorem. The notion of rationalizability allows for the possibility that an agent's observed choices at a given feasible set constitute some, but not all, of her optimal choices. As we pointed out in Section 2, for this notion to be nonvacuous, we must impose further constraints on the agent's choice behavior, such as being consistent with an exogeneously given dominance relation, that would preclude her being indifferent across all alternatives. On the other hand, in economic contexts where such a relation is not naturally present, or when the observer has reasons to believe that the unchosen alternatives in a feasible set are strictly less preferred to the ones chosen, it may be appropriate to adopt a stronger notion of rationalizability. 
Given a choice environment $((X, \unrhd), \mathcal{A})$ and a choice correspondence $c$ on $\mathcal{A}$, we say that a preference relation $\succsim$ on $X$ strictly rationalizes $c$ if

$$
c(A)=\max (A, \succsim)
$$

for every $A \in \mathcal{A}$. If, in addition, $\succsim$ extends the preorder $\unrhd$, we say that it strictly $\unrhd$-rationalizes $c$. If $X$ is a topological space and there is a continuous and strictly $\unrhd$-increasing function $u: X \rightarrow \mathbb{R}$ such that, for every $A \in \mathcal{A}$,

$$
c(A)=\arg \max \{u(x): x \in A\}
$$

then we say that $c$ is strictly $\unrhd$-rationalizable by a continuous utility function.

While it may sometimes be too demanding in the context of empirical studies, the notion of strict rationalizability of a choice correspondence is widely studied in (rational or boundedly rational) choice theory, and the property is understood as providing a theoretical foundation for the utility maximization paradigm. (Classical references using this concept include Samuelson (1938), Houthakker (1950), and Arrow (1959).) One of the major results on strict rationalizability is found in the seminal work of Richter (1966). This result is based on a property called the congruence axiom; a choice correspondence $c$ on $\mathcal{A}$ satisfies this axiom if

$$
x \operatorname{tran}(R(c)) y \text { and } y \in c(A) \quad \text { imply } \quad x \in c(A)
$$

for every $A \in \mathcal{A}$ that contains $x$. In other words, whenever a feasible alternative $x$ in a choice problem is revealed preferred to an alternative $y$ that is chosen in that problem, then $x$ must be chosen in that problem as well. Richter's Theorem says that a choice correspondence $c$ on $\mathcal{A}$ is rationalizable iff it satisfies the congruence axiom.

4.2. The congruence axiom in general choice environments. In the context of an arbitrarily given choice environment $((X, \unrhd), \mathcal{A})$, we would like to generalize Richter's Theorem by characterizing all choice correspondences on $\mathcal{A}$ that are strictly $\unrhd$-rationalizable. Clearly, this calls for a suitable generalization of Richter's congruence axiom, so we begin our work in this section by introducing such a generalization: a choice correspondence $c$ on $\mathcal{A}$ is said to satisfy the $\unrhd$-congruence axiom if

$$
x \operatorname{tran}(R(c) \cup \unrhd) y \quad \text { and } \quad y \in c(A) \quad \text { imply } \quad x \in c(A)
$$

for every $A \in \mathcal{A}$ that contains $x$, and

$$
x \operatorname{tran}(R(c) \cup \unrhd) y \quad \text { implies } \quad \operatorname{not} y \triangleright x
$$


for every $x, y \in X$. This is a bonafide generalization of the standard congruence axiom. Indeed, if we take $\unrhd$ as the partial order $\triangle_{X}:=\{(x, x): x \in X\}$ (which says that there is no dominance between any two distinct alternatives), then this definition coincides with that of the standard congruence axiom.

It follows immediately from Proposition 1 that the $\unrhd$-congruence axiom is stronger than cyclical $\unrhd$-consistency. Our next proposition reformulates $\unrhd$-congruence in a way that makes the relationship between the two properties even clearer.

Proposition 6. Let $((X, \unrhd), \mathcal{A})$ be a choice environment. Then, a choice correspondence $c$ on $\mathcal{A}$ satisfies the $\unrhd$-congruence axiom if, and only if, for every integer $k \geq 2$ and $A_{1}, \ldots, A_{k} \in \mathcal{A}$,

$$
x_{1} \in c\left(A_{1}\right) \cap A_{2}^{\downarrow}, \ldots, x_{k-1} \in c\left(A_{k-1}\right) \cap A_{k}^{\downarrow} \text { and } x_{k} \in c\left(A_{k}\right) \cap A_{1}^{\downarrow}
$$

imply (4) as well as

$$
x_{1}^{\uparrow} \cap A_{2} \subseteq c\left(A_{2}\right), \ldots, x_{k-1}^{\uparrow} \cap A_{k} \subseteq c\left(A_{k}\right) \text { and } x_{k}^{\uparrow} \cap A_{1} \subseteq c\left(A_{1}\right) \cdot{ }^{21}
$$

Thus, while cyclical $\unrhd$-consistency requires that (3) implies (4), the $\unrhd$-congruence axiom requires that (3) implies (4) and (12). It follows from Proposition 6 that the four-step procedure set out in Section 3.5 for checking cyclical $\unrhd$-consistency can also be used to check for the $\unrhd$-congruence axiom, provided that we modify Step IV in that procedure to check that properties (4) and (12) hold for every cycle in $\mathcal{S}$.

4.3. Generalizing Richter's Theorem. The following result shows that $\unrhd$-congruence axiom is necessary and sufficient for the strong $\unrhd$-rationalizability of any choice correspondence in any choice environment.

Theorem 7. Let $((X, \unrhd), \mathcal{A})$ be a choice environment. Then, a choice correspondence $c$ on $\mathcal{A}$ satisfies the $\unrhd$-congruence axiom if, and only if, it is strictly $\unrhd$-rationalizable.

Theorem 7 is best viewed as a monotone version of Richter's Theorem, but it is worth noting that this result is a proper generalization of Richter's Theorem. Indeed, setting $\unrhd$ to be the preorder $\triangle_{X}$ in Theorem 7 , we recover the exact statement of Richter's Theorem. Given the groundwork we have laid out so far, this result has a short proof and, in particular, the sufficiency of the $\unrhd$-congruence axiom for strict rationalizability follows easily from Proposition 5. That proposition says that when $c$ obeys cyclical $\unrhd$ consistency, there is a preference relation $\succsim$ that not only $\unrhd$-rationalizes $c$ but has the

\footnotetext{
${ }^{21}$ For any $x$ in $X$, we let $x^{\uparrow}$ stand for the increasing closure of $\{x\}$ with respect to $\unrhd$, that is, $x^{\uparrow}:=\{y \in X: y \unrhd x\}$.
} 
added property that (for all $A \in \mathcal{A}$ ) the set $\max (A, \succsim)$ is minimal, even though it may be a strict superset of $c(A)$. In fact, one could go on to show that when $c$ obeys the stronger property of $\unrhd$-congruence, the two sets will coincide for all $A \in \mathcal{A}$.

Just like we have done in the case of our extensions of Afriat's Theorem, and for the same motivating reasons, we would like to upgrade Theorem 7 to obtain a monotone and continuous version of Richter's Theorem. This following result covers this case.

Theorem 8. Let $((X, \unrhd), \mathcal{A})$ be a continuous choice environment such that $\mathcal{A}$ is a finite set. Then, a closed-valued choice correspondence $c$ on $\mathcal{A}$ satisfies the $\unrhd$-congruence axiom if, and only if, it is strictly $\unrhd$-rationalizable by a continuous utility function.

4.4. Strict rationalizability in classical choice environments. If only to provide a quick application, we now return to the classical Afriat model, and investigate what it would take for the observed consumption demand data to be strictly rationalizable in that model. To this end, take a nonempty finite set $P$ of price vectors in $\mathbb{R}_{++}^{n}$, and let $d: P \rightrightarrows \mathbb{R}^{n}$ be a correspondence such that $d(\mathbf{p})$ is a nonempty subset of $\mathbb{R}_{+}^{n} \backslash\{\mathbf{0}\}$ with $\mathbf{p} d(\mathbf{p})=1$, for all $\mathbf{p} \in P$. (Recall that in Section 3.1 we refer to this map as a (normalized) demand correspondence on $P$.) $P$ induces a classical consumption choice environment $\left(\left(\mathbb{R}_{+}^{n}, \geq\right), \mathcal{A}\right)$, where $\mathcal{A}:=\{B(\mathbf{p}, 1): \mathbf{p} \in P\}$ and with $d$ we can define the choice correspondence $c$ on $\mathcal{A}$ by $c(B(\mathbf{p}, 1)):=d(\mathbf{p})$. . We say that $d$ satisfies the $\geq$-congruence (or congruence) axiom, or that $d$ is strictly $\geq$-rationalizable by a continuous utility function, if $c$ possesses the corresponding properties, respectively.

Since $\mathbf{p} d(\mathbf{p})=1$ for all $\mathbf{p} \in P$, there is no $\mathbf{y}$ in $B(\mathbf{p}, 1)$ with $\mathbf{y}>\mathbf{x}$ for any $x$ in $B(\mathbf{p}, 1)$. Furthermore, $B(\mathbf{p}, 1)^{\downarrow}=B(\mathbf{p}, 1)$, so $\mathbf{x} \operatorname{tran}(R(c) \cup \geq) \mathbf{y}$ iff $\mathbf{x} \operatorname{tran}(R(c)) \mathbf{y}$. Given these observations, one readily checks that $d$ satisfies the $\geq$-congruence axiom iff it satisfies the congruence axiom. Thus, Theorem 8 yields the following result.

Proposition 9. Let $P$ be a nonempty finite set in $\mathbb{R}_{++}^{n}$ and $d$ a closed-valued (normalized) demand correspondence on $P$. Then $d$ is strictly $\geq$-rationalizable by a continuous utility function if, and only if, it satisfies the congruence axiom.

We note that Proposition 9 is related to a theorem of Matzkin and Richter (1991), which also provides a characterization of strict rationalizability of $d$, but under the hypothesis that $d$ is single-valued. The rationalizing preference relation on $\mathbb{R}_{+}^{n}$ obtained by Matzkin and Richter is representable by a continuous, strictly increasing, and strictly concave utility function on $\mathbb{R}_{+}^{n}$. In our case, the last property is not guaranteed: if $d$ is not convex-valued, a preference relation that strictly rationalizes $d$ cannot be convex, and hence, no utility function that represents this relation can be even quasiconcave. 


\section{FURTHER APPLICATIONS}

The main theorems of Sections 3 and 4 provide methods for studying (strict) rationalizability in choice environments well beyond the examples we have covered so far. In this section, we provide further applications of our results in several economically important and widely-studied choice environments.

5.1. Choice over policy space. In formal political science, it is common to model political positions as points in a Euclidean space $\mathbb{R}_{+}^{n}$, where $n$ is interpreted as the number of issues over which a policymaker may form a preference. However, in this context, it is no longer sensible to require individual preferences to extend the coordinate-wise order $\geq$; indeed the policymaker may well have an ideal position in $\mathbb{R}_{+}^{n}$ that is strictly preferred to all other alternatives. If the observer has no further knowledge about the policymaker's preferences, then this is captured by requiring $\unrhd$ to equal $\Delta_{\mathbb{R}^{n}}$. The choice environment in thus $\left(\left(\mathbb{R}^{n}, \Delta_{\mathbb{R}^{n}}\right), \mathcal{A}\right)$, where we assume that $\mathcal{A}$ is a nonempty finite collection of compact subsets of $\mathbb{R}_{+}^{n}$. Suppose, in addition, that the policymaker's observed choices is given by some closed-valued correspondence $c$ on $\mathcal{A}$. In this setting, there is no nontrivial test for rationalizability, but it still possible to test for strict rationalizability. Theorem 8 tells us that $c$ is strictly rationalizable by a continuous utility function on $\mathbb{R}_{+}^{n}$ iff it satisfies the congruence axiom. ${ }^{22}$

Suppose one makes the further hypothesis that the policymaker will always agree with the position of a certain group of agents $\mathcal{I}$; this can be formally captured by requiring the policymaker's preference relation on $\mathbb{R}_{+}^{n}$ to extend a (not necessarily complete) preorder $\gtrsim^{\mathcal{I}}$ on $\mathbb{R}_{+}^{n}$ (which we interpret as group $\mathcal{I}$ 's policy preferences and which we assume is observable). ${ }^{23}$ Theorem 3 says that, provided $\gtrsim^{\mathcal{I}}$ is continuous, $\mathcal{A}$ is a finite collection of compact sets, and $c$ is closed-valued, the following holds: there is a continuous utility function that $\gtrsim^{\mathcal{I}}$-rationalizes $c$ iff it obeys cyclical $\gtrsim^{\mathcal{I}}$-consistency.

5.2 Recoverability. In this application, we turn again to classical consumption choice environments. As in Section 4.4, take a nonempty finite set $P$ of price vectors in $\mathbb{R}_{++}^{n}$, and let $d$ stand for the (normalized) demand correspondence on $P$. Suppose $d$ satisfies cyclical consistency, and denote by $\mathcal{U}(d)$ the collection of all continuous utility functions

\footnotetext{
${ }^{22}$ Kalandrakis (2010) provides a different revealed preference analysis in a similar setting. In our terminology, Kalandrakis considers the case where each $A \in \mathcal{A}$ has two elements, and characterizes those single-valued choice correspondences on $\mathcal{A}$ that can be rationalized by a concave (hence continuous) utility function on $\mathbb{R}^{n}$. Our rationalization imposes weaker assumptions on $\mathcal{A}$ and $c$, and the rationalizing utility function we obtain, while continuous, need not be concave. (Such nonconcavities may arise naturally in policy spaces; see, for example, Stiglitz (1974).)

${ }^{23}$ For example, we may have $\gtrsim^{\mathcal{I}}=\cap_{i \in \mathcal{I}} \gtrsim_{i}$, where $\gtrsim_{i}$ is the member $i$ 's preference relation on $\mathbb{R}_{+}^{n}$.
} 
$u: \mathbb{R}_{+}^{n} \rightarrow \mathbb{R}$ that $\geq$-rationalize $d$. (By Theorem $3, \mathcal{U}(d) \neq \varnothing$.) Since different members of $\mathcal{U}(d)$ will have different preference rankings across bundles, it would be useful to find out the extent to which we may identify the actual preference relation of the agent without subscribing to any one utility function that $\geq$-rationalizes $d$. Formally, we would like to characterize the subsets $S(d)$ and $S^{\prime}(d)$ of $\mathbb{R}_{+}^{n} \times \mathbb{R}_{+}^{n}$ such that

$$
(\mathbf{x}, \mathbf{y}) \in S(d) \quad \text { iff } \quad u(\mathbf{x}) \geq u(\mathbf{y}) \text { for each } u \in \mathcal{U}(d)
$$

and

$$
(\mathbf{x}, \mathbf{y}) \in S^{\prime}(d) \quad \text { iff } \quad u(\mathbf{x})>u(\mathbf{y}) \text { for each } u \in \mathcal{U}(d)
$$

This formulation of the problem is the same as what Varian (1982) calls the Recoverability Problem, except that Varian considers the case where $\mathcal{U}(d)$ consists of continuous and concave utility functions that $\geq$-rationalizes $d$. Given the weaker assumptions on the utility functions we impose here, the relations $S(d)$ and $S^{\prime}(d)$ are bound to be smaller than those studied by Varian (1982). Moreover, in certain contexts, it is sensible not to impose a concavity, or even quasiconcavity, requirement on the utility functions (even when it is possible to rationalize $d$ by such a utility function). To wit, consider a situation in which the consumer chooses a contingent consumption over $n$ states of the world, subject to a linear budget set, where a bundle $d(\mathbf{p})$ is the bundle demanded at the state prices p. In such a context, as also noted by Halevy et al. (2014), we may well wish to draw inferences of the agent's preferences (based on her observed choices) without assuming that she has a quasiconcave utility function, since that assumption would exclude risk-seeking and/or elation-seeking preferences. ${ }^{24}$

For bundles $\mathbf{x}$ and $\mathbf{y}$ in $\mathbb{R}_{+}^{n}$, we say that $\mathbf{x}$ is revealed preferred to $\mathbf{y}$ if $\mathbf{x} \operatorname{tran}(R(c) \cup$ $\geq) \mathbf{y}$, where, as usual, $c$ is the choice correspondence induced by $d$ (Section 4.4). This is equivalent to saying that there are finitely many $\mathbf{p}^{1}, \ldots, \mathbf{p}^{k} \in P$ and $\mathbf{x}^{1} \in d\left(\mathbf{p}^{1}\right), \ldots, \mathbf{x}^{k} \in$ $d\left(\mathbf{p}^{k}\right)$ such that

$$
\mathbf{x} \geq \mathbf{x}^{1}, \mathbf{p}^{1} \mathbf{x}^{1} \geq \mathbf{p}^{1} \mathbf{x}^{2}, \ldots, \mathbf{p}^{k-1} \mathbf{x}^{k-1} \geq \mathbf{p}^{k-1} \mathbf{x}^{k} \text { and } \mathbf{p}^{k} \mathbf{x}^{k} \geq \mathbf{p}^{k} \mathbf{y}
$$

We say that $\mathbf{x}$ is revealed strictly preferred to $\mathbf{y}$ if any of the inequalities in (13) is strict. It is clear that if $\mathbf{x}$ is revealed (strictly) preferred to $\mathbf{y}$, then $(\mathbf{x}, \mathbf{y})$ belongs to $S(d)$ (respectively, $S^{\prime}(d)$ ). Our next result, whose proof relies on Proposition 5 and

\footnotetext{
${ }^{24}$ For example, suppose that $n=2$ and the consumer's true utility function is $u\left(x_{1}, x_{2}\right)=\pi_{1} v\left(x_{1}\right)+$ $\pi_{2} v\left(x_{2}\right)$, where $\pi_{i}>0$ for $i=1,2$, and $v$ is strictly increasing but not concave. Then $u$ will not be quasiconcave and predicting the consumer's preference from $d$ while assuming quasiconcavity can lead to false conclusions. On the other hand, the predictions captured by $S(d)$ and $S^{\prime}(d)$ will be correct.
} 
Theorem 3, shows that the converse is also true, thereby yielding a practical method of computing the preorders $S(d)$ and $S^{\prime}(d)$.

Proposition $10 .^{25}$ Let $P$ be a nonempty finite set in $\mathbb{R}_{++}^{n}$ and $d$ a closed-valued (normalized) demand correspondence on $P$ which satisfies cyclical $\geq$-consistency. Then,

(i) $(\mathbf{x}, \mathbf{y}) \in S(d)$ if, and only if, $\mathbf{x}$ is revealed preferred to $\mathbf{y}$; and

(ii) $(\mathbf{x}, \mathbf{y}) \in S^{\prime}(d)$ if, and only if, $\mathbf{x}$ is revealed strictly preferred to $\mathbf{y}$.

5.3 Rationalizability of Engel curves. Consider a researcher who has estimated the Engel curves at a finite set of price vectors. When is it the case that these curves correspond to those of a decision maker maximizing a continuous and strictly increasing utility function? This rationalizability problem cannot be directly addressed by Afriat's Theorem, since even a single Engel curve presumes uncountably many choice situations. ${ }^{26}$ We can, however, provide an answer fairly easily by appealing to Theorem 4. To be precise, the choice environment is of the form $\left(\left(\mathbb{R}_{+}^{n}, \geq\right), \mathcal{A}\right)$, where

$$
\mathcal{A}:=\{B(\mathbf{p}, I): \mathbf{p} \in P \text { and } I \in \mathbb{I}\}
$$

with $P$ being a nonempty finite subset of $\mathbb{R}_{++}^{n}$ and $\mathbb{I}$ being a nondegenerate closed interval in $\mathbb{R}_{+}$. We are given a demand correspondence of the form $d: P \times \mathbb{I} \rightrightarrows \mathbb{R}_{+}^{n}$ such that $\mathbf{p x}=I$ for every $\mathbf{x} \in d(\mathbf{p}, I)$ and $(\mathbf{p}, I) \in P \times \mathbb{I}$. (The map $I \mapsto d(B(\mathbf{p}, I))$ is the Engel curve of the agent at the price vector p.) Abusing the terminology, we say that $d$ (or the Engel curves induced by $d$ ) satisfies cyclical $\geq$-consistency or the congruence axiom if the choice correspondence $c: B(\mathbf{p}, I) \mapsto d(\mathbf{p}, I)$ on $\mathcal{A}$ satisfies the corresponding property.

This is essentially the setup of Blundell et al. (2003), who estimated Engel curves on a finite set of price vectors and then tested if these curves satisfied cyclical $\geq$-consistency. Of course, the Engel curves of a utility maximizing consumer must obey cyclical $\geq$ consistency, but empirical welfare analysis based on data that satisfies this property (such as the one carried out by Blundell et al. (2003)) presumes that it is also sufficient for rationalizability. This presumption is by no means self-evident, but the following result shows that it is nevertheless correct.

\footnotetext{
${ }^{25}$ If we do not require $P$ to be finite or $d$ to be closed-valued, then so long as $d$ obeys cyclical consistency we obtain the following: (i) $\mathbf{x}$ is revealed preferred to $\mathbf{y}$ iff $x \succsim y$ for each preference $\succsim$ that $\geq$-rationalizes $d$ and (ii) $\mathbf{x}$ is strictly revealed preferred to $\mathbf{y}$ iff $x \succ y$ for every preference $\succsim$ that $\geq$-rationalizes $d$. (But, in this case, preferences need not be continuous.)

${ }^{26}$ While the rationalizability theorem of Reny (2015) applies to infinite data sets that satisfy cyclical consistency, the utility function it constructs is in general not continuous.
} 
Proposition 11. Let $\left(\left(\mathbb{R}_{+}^{n}, \geq\right), \mathcal{A}\right)$ be the choice environment defined by $(14)$, and let $d: P \times \mathbb{I} \rightrightarrows \mathbb{R}_{+}^{n}$ be an upper hemicontinuous demand correspondence. Then there exists a continuous utility function $u: \mathbb{R}_{+}^{n} \rightarrow \mathbb{R}$ that (i) $\geq$-rationalizes $d$ if, and only if, $d$ satisfies cyclical $\geq$-consistency, and (ii) strictly $\geq$-rationalizes $d$ if, and only if, $d$ satisfies the $\geq$-congruence axiom. ${ }^{27}$

As $\mathcal{A}$ is not finite here, we cannot make use of Theorem 3 to prove this proposition. Instead, our proof uses the upper hemicontinuity of $d$ to ensure that $\operatorname{tran}(R(c) \cup \geq)$ is a continuous preorder on $\mathbb{R}_{+}^{n}$, where $c$ is the choice correspondence on $\mathcal{A}$ induced by $d$. This allows us to use Theorem 4 to obtain a continuous utility function that (strictly) $\geq$-rationalizes $d$ as asserted in Proposition 11 .

5.4 Contingent consumption. Suppose there are $n$ states of the world, where the probability of each state $i$, which we denote by $\pi_{i}$, is known to both the consumer and the observer. The consumer chooses a bundle of contingent consumption subject to a linear budget set at a given vector of state prices. In such a context, Varian (1983) and Green and Srivastava (1986), among others, have developed characterizations of data sets consistent with the hypothesis that the consumer maximizes an expected utility function. Our objective here is to develop a test for a more permissive model of rational behavior. We ask if the agent's behavior is consistent with the maximization of a continuous utility function that is strictly increasing with respect to first order stochastic dominance. ${ }^{28}$

In formal terms, we consider the choice environment $\left(\left(\mathbb{R}_{+}^{n}, \geq_{\mathrm{FSD}, \pi}\right), \mathcal{A}\right)$, where $P$ is a nonempty finite subset of $\mathbb{R}_{++}^{n}, \mathcal{A}:=\{B(\mathbf{p}, 1): \mathbf{p} \in P\}$, and $\geq_{\mathrm{FSD}, \pi}$ is the first order stochastic dominance relation on $\mathbb{R}_{+}^{n}$ induced by the probability vector $\pi:=\left(\pi_{1}, \ldots, \pi_{n}\right){ }^{29}$ Note that the binary relation $\geq_{\mathrm{FSD}, \pi}$ is a continuous preorder on $\mathbb{R}_{+}^{n}$, but, unlike the first order stochastic dominance relation we worked with in Example 3 in Section 3.4, it is not a partial order. (For instance, $(1,2)$ and $(2,1)$ are equivalent with respect to $\gtrsim_{\text {FSD },\left(\frac{1}{2}, \frac{1}{2}\right)}$.) Now, for any closed-valued (normalized) demand correspondence $d$ on $P$, Theorem 3 says that $d$ is $\geq_{\mathrm{FSD}, \pi}$-rationalizable by a continuous utility function $u: \mathbb{R}_{+}^{n} \rightarrow \mathbb{R}$ iff it obeys cyclical $\geq_{\mathrm{FSD}, \pi}$-consistency. ${ }^{30}$ To actually implement such a test on data, one

\footnotetext{
${ }^{27}$ For an empirical implementation of the test for cyclical consistency on Engel curves see Blundell et al. (2003).

${ }^{28}$ Easy examples show that it is possible for a preference to be representable by utility function that is strictly $\gtrsim_{F S D, \pi}$-increasing, without being representable by an expected utility function.

${ }^{29}$ For any $n$-vector $\mathbf{z}=\left(z_{1}, z_{2}, \ldots, z_{n}\right)$ and real number $a \geq 0$, put $I(\mathbf{z}, a):=\left\{i: z_{i} \leq a\right\}$. Then, for any nonnegative $n$-vectors $\mathbf{x}$ and $\mathbf{y}$, we have $\mathbf{x} \geq_{\mathrm{FSD}, \pi} \mathbf{y}$ iff $\sum_{i \in I(\mathbf{x}, a)} \pi_{i} \leq \sum_{I(\mathbf{y}, a)} \pi_{i}$ for each $a \geq 0$.

${ }^{30}$ Since $>_{\mathrm{FSD}, \pi} \supset>$ (where $>_{\mathrm{FSD}, \pi}$ and $>$ are asymmmetric parts of $\geq_{\mathrm{FSD}, \pi}$ and $\geq$ respectively), $d$ is $\geq$-rationalizable whenever it is $\geq_{\mathrm{FSD}, \pi}$-rationalizable.
} 
could use the four-step procedure set out in Section 3.5. ${ }^{31}$

5.5. Intertemporal consumption. Consider a researcher who observes a consumer choosing consumption of a single good across $n$ different future dates, subject to a given wealth and the price of consumption at different dates. A consumption stream in this framework is an element $\mathbf{x}$ of $\mathbb{R}_{+}^{n}$ and the price vector is an element $\mathbf{p}$ of $\mathbb{R}_{++}^{n}$. (For an experiment where subjects are observed to make choices in such a setting, see Andreoni and Sprenger (2012).) To avoid issues that may arise due to dynamic inconsistency, we assume that the analyst observes the commitment choices of the agent, that is, the consumer cannot deviate from her chosen consumption stream. ${ }^{32}$

In the study of intertemporal consumption, a question that has received quite a lot of attention is whether consumers display impatience. This property is a feature of geometric discounting, even though there is considerable evidence challenging its empirical prevalence. (See, for instance, Frederick et al. (2002) for a survey.) There are revealed preference tests that specifically checks for consistency with the geometric discounting model (cf. Browning (1989) and Echenique et al. (2015)), but it would also be interesting to test whether the consumer's behavior exhibits impatience, whether or not it satisfies geometric discounting. Our results can be used to devise such tests.

Let us first consider the case where the consumer, instead of being impatient, is indifferent about the timing of consumption. Formally, an intertemporal utility function $U: \mathbb{R}_{+}^{n} \rightarrow \mathbb{R}$ exhibits neutral time preference if it is strictly increasing and symmetric. (By symmetry of $U$ here, we mean that $U(\mathbf{x})=U\left(\mathbf{x}_{\sigma}\right)$ for any $\mathbf{x} \in \mathbb{R}_{+}^{n}$ and any permutation $\sigma$ on $N:=\{1,2, \ldots, n\}$, where $\mathbf{x}_{\sigma}$ stands for the $n$-vector $\left(z_{\sigma(1)}, \ldots, z_{\sigma(n)}\right)$.) In turn, we define the binary relation $\gtrsim_{\text {sym }}$ on $\mathbb{R}_{+}^{n}$ by $\mathbf{x} \gtrsim_{\text {sym }} \mathbf{y}$ if $\mathbf{x} \geq \mathbf{y}_{\sigma}$ for some permutation $\sigma$ on $N$. Obviously, a person who is indifferent over the timing of consumption would be indifferent between $\mathbf{y}$ and $\mathbf{y}_{\sigma}$ (for any $\sigma$ ), so, assuming that she prefers more to less, she would prefer $\mathbf{x}$ over $\mathbf{y}$ whenever $\mathbf{x} \gtrsim_{\text {sym }} \mathbf{y}$. It is readily verified that $\gtrsim_{\text {sym }}$ is a continuous preorder on $\mathbb{R}_{+}^{n}$ (but it is not a partial order unless $n=1$ ). Moreover, an intertemporal utility function $U: \mathbb{R}_{+}^{n} \rightarrow \mathbb{R}$ exhibits a neutral time preference iff it is strictly

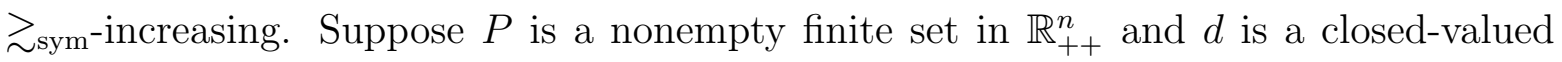
(normalized) demand correspondence on P. By Theorem 3, $d$ is $\gtrsim$ sym-rationalizable by a continuous utility function iff it satisfies cyclical $\gtrsim_{\text {sym-consistency. }}$

\footnotetext{
31 Polisson et al. (2015) have recently implemented this test procedure on the experimental data collected by Choi et al. (2007). See also the related tests carried out by Heufer (2014) on this data set.

32 There is a related strand of revealed preference studies where observations consist of choices made over consumption-date pairs (rather than consumption streams); see, for example, Demuynck (2009) and Dziewulski (2015).
} 
To test for impatience, we would require the consumer's utility function to be strictly increasing with respect to a different preorder. Given any $\mathbf{x} \in \mathbb{R}_{+}^{n}$, let us call an $n$-vector $\mathbf{y}$ an improving swap of $\mathbf{x}$ if $\mathbf{y}=\mathbf{x}_{\sigma}$ for some permutation $\sigma$ on $N$ such that there exist $k$ and $l$ in $N$ with $k<l, x_{k} \leq x_{l}, \sigma(k)=l, \sigma(l)=k$ and $\sigma(j)=j$ for all $j \in N \backslash\{k, l\}$. (For example, $(4,3,6,5)$ is an improving swap of $(4,3,5,6)$.) The bundle $\mathbf{y}$ is an improving permutation of $\mathbf{x}$ if $\mathbf{y}$ is obtained from $\mathbf{x}$ by means of a sequence of improving swaps. Lastly, we define the binary relation $\gtrsim_{+}$on $\mathbb{R}_{+}^{n}$ by setting $\mathbf{x} \gtrsim_{+} \mathbf{y}$ whenever there is a vector $\mathbf{z}$, which is an improving permutation of $\mathbf{y}$, such that $\mathbf{x} \geq \mathbf{z}$. (Clearly, $\gtrsim_{+}$is a continuous preorder on $\mathbb{R}_{+}^{n}$.) We say that an intertemporal utility function $U: \mathbb{R}_{+}^{n} \rightarrow \mathbb{R}$ exhibits positive time preference if it is strictly $\gtrsim_{+}$-increasing. ${ }^{33}$ Once again, by appealing to Theorem 3 , we see that cyclical $\gtrsim_{+}$-consistency characterizes those (normalized) demand correspondences that are rationalizable by continuous utility functions with positive time preference.

\section{APPENDIX}

Proof of Proposition 1. Let $c$ be a choice correspondence on $\mathcal{A}$ such that (5) holds for every $x, y \in X$. Take any integer $k \geq 2$ and $A_{1}, \ldots, A_{k} \in \mathcal{A}$. Pick any $x_{1}, \ldots, x_{k} \in X$ with

$$
x_{i} \in c\left(A_{i}\right) \cap A_{i+1(\bmod k)}^{\downarrow} \text { for each } i=1, \ldots, k .
$$

Then, for every $i$, there is a $y_{i+1}(\bmod k) \in A_{i+1}(\bmod k)$ such that $x_{i+1}(\bmod k) R(c) y_{i+1}(\bmod k) \unrhd$ $x_{i}$. It follows that

$$
x_{k} \operatorname{tran}(R(c) \cup \unrhd) x_{k-1} \operatorname{tran}(R(c) \cup \unrhd) \cdots \operatorname{tran}(R(c) \cup \unrhd) x_{1} .
$$

Now take any $y \in A_{1}^{\downarrow}$ such that $y \unrhd x_{k}$. Then, $x_{1} \operatorname{tran}(R(c) \cup \unrhd) y$, so the previous observation yields $x_{k} \operatorname{tran}(R(c) \cup \unrhd) y$. By (5), therefore, $y \triangleright x_{k}$ cannot hold. In view of the arbitrary choice of $y$ in $A_{1}^{\downarrow}$, we may thus conclude that $x_{k}$ does not belong to $A_{1}^{\downarrow \downarrow}$. That $x_{i}$ does not belong to $A_{i+1}^{\downarrow}$ is analogously established for each $i=1, \ldots, k-1$. Thus $c$ satisfies cyclical $\unrhd$-consistency.

Conversely, assume that $c$ is a choice correspondence on $\mathcal{A}$ which satisfies cyclical $\unrhd$ consistency. Suppose $x \operatorname{tran}(R(c) \cup \unrhd) y$ but $y \triangleright x$. Then, there exist $x_{1}, \ldots, x_{k}$ and $y_{1}, \ldots, y_{k}$ in $X$ such that

$$
x \unrhd x_{k} R(c) y_{k} \unrhd x_{k-1} R(c) \cdots \unrhd x_{2} R(c) y_{2} \unrhd x_{1} R(c) y_{1} \unrhd y \triangleright x \unrhd x_{k} .
$$

\footnotetext{
${ }^{33}$ For instance, suppose $U$ is defined by $U(\mathbf{x}):=\sum_{i=1}^{n} v\left(i, \mathbf{x}_{i}\right)$, where $v$ is strictly decreasing in this its first component, and strictly increasing in the second. Then $U$ exhibits positive time preference. Note that any function that is strictly $\gtrsim_{+}$-increasing must also be strictly $\geq$-increasing.
} 
Since $x_{i} R(c) y_{i}$, there is an $A_{i} \in \mathcal{A}$ such that $\left(x_{i}, y_{i}\right) \in c\left(A_{i}\right) \times A_{i}$, for each $i=1, \ldots, k$. Therefore, (15) implies that $x_{i} \in c\left(A_{i}\right) \cap A_{i+1(\bmod k)}^{\downarrow}$ for each $i=1, \ldots, k$. By cyclical $\unrhd$ consistency of $c$, then, $x_{k}$ does not belong to $A_{1}^{\downarrow \downarrow}$, but this is false because $y_{1} \in A_{1}$ and $y_{1} \triangleright x_{k}$.

Proof of Theorem 2. We have already proved at the beginning of Section 3.3 that any choice correspondence that obeys cyclical $\unrhd$-consistency is $\unrhd$-rationalizable. Conversely, assume that $c$ is a choice correspondence on $\mathcal{A}$ that satisfies cyclical $\unrhd$-consistency. Define $\succsim^{*}:=\operatorname{tran}(R(c) \cup \unrhd$ ), which is a preorder on $X$. By Szpilrajn's Theorem, there is a complete preorder $\succsim$ on $X$ that extends $\succsim^{*}$. As $R(c) \subseteq \succsim$, we have $x \succsim y$ if there is an $A \in \mathcal{A}$ with $(x, y) \in c(A) \times A$. It follows that $c(A) \subseteq \max (A, \succsim)$ for every $A \in \mathcal{A}$. It remains to show that $\succsim$ extends $\unrhd$, and for this, it is enough to show that $\triangleright \subseteq \succ^{*}$. To this end, take any two elements $x$ and $y$ of $X$ such that $x \triangleright y$. By definition of $\succsim^{*}$, we have $x \succsim^{*} y$. Furthermore, $y \succsim^{*} x$ cannot hold since, otherwise, Proposition 1 would imply that $x \triangleright y$ is false. We thus have $x \succ^{*} y$, as we sought.

Our proofs of Theorems 3 and 4 are built on the following result of topological order theory.

Levin's Theorem. Let $X$ be a locally compact and separable metric space and $\succsim$ a continuous preorder on $X$. Then there is a preference relation on $X$ that extends $\succsim$ and admits a continuous utility representation.

This result, which we refer to here as Levin's Theorem, is actually a special case of the main theorem of Levin (1983), who only assumed that $X$ is a locally compact, $\sigma$-compact and second countable Hausdorff space. It is well-known in topological order theory; see, for instance, Herden (1989), Bridges and Mehta (1996), and Herden and Pallack (2002). Given that this theorem is a main building block for some of the central results of this paper, we would like to provide here a fairly direct and accessible proof, based on the following better known theorem.

The Nachbin Extension Theorem. ${ }^{34}$ Let $X$ be a compact Hausdorff space, and $Y$ a nonempty closed subset of $X$. Let $\succsim$ be a continuous preorder on $X$. If $f$ is a continuous and $\succsim$-increasing real map on $Y$, then there is a continuous and $\succsim$-increasing real map $F$ on $X$ with $\left.F\right|_{Y}=f .{ }^{35}$

We now show how one may easily derive Levin's Theorem from this result. (Levin's original proof was fairly long, and did not make use of Nachbin's Theorem.)

Lemma A. Let $X$ be a compact metric space, and $\succsim$ a continuous preorder on $X$. Then, there is a continuous and strictly $\succsim$-increasing map $U: X \rightarrow[0,1]$.

\footnotetext{
34 This result obtains as a consequence of putting together Theorems 4 and 6 of Chapter 1 of Nachbin (1965). (Nachbin actually assumes that $\succsim$ is a partial order on $X$ in these results, but so long as $X$ is compact and Hausdorff, his proofs apply (without modification) to the case of preorders as well.)

${ }^{35}$ Note that neither $f$ nor $F$ is strictly $\succsim$-increasing in this theorem.
} 
Proof of Lemma A. Define $\Omega:=\{(x, y) \in X \times X:$ not $x \succsim y\}$. If $\Omega=\emptyset$, then $x \sim y$ for all $x, y \in X$, so any constant real map on $X$ fulfils the requirements of the lemma. We assume, then, $\Omega \neq \emptyset$. In turn, for any $(x, y) \in \Omega$, we define the function $v_{x, y}:\{x, y\} \rightarrow \mathbb{R}$ as $v_{x, y}(x):=0$ and $v_{x, y}(y):=1$, which is a continuous and $\succsim$-increasing function on $\{x, y\}$. By the Nachbin Extension Theorem, for each $(x, y) \in \Omega$ there is a continuous and $\succsim$-increasing function $u_{x, y}: X \rightarrow \mathbb{R}$ such that $u_{x, y}(x)=0$ and $u_{x, y}(y)=1$; we denote the collection of these functions by $\mathcal{U}$. Then, it is readily checked that

$$
x \succsim y \text { iff } u(x) \geq u(y) \text { for every } u \in \mathcal{U}
$$

for every $x, y \in X$. In what follows, we assume, without loss of generality, that each $u \in \mathcal{U}$ is $[0,1]$-valued. (Otherwise, we would have replaced each $u$ in $\mathcal{U}$ with, say, $\frac{1}{\pi} \arctan u+\frac{1}{2}$.)

As usual, we let $\mathbf{C}(X)$ stand for the set of all continuous real functions on $X$, and metrize this set by means of the sup-metric. Since $X$ is compact, $\mathbf{C}(X)$ is a separable metric space. As a metric subspace of a separable metric space is separable, therefore, there is a countable subset $\mathcal{V}$ of $\mathcal{U}$ which is dense in $\mathcal{U}$. Now, take any elements $x$ and $y$ of $X$. Obviously, if $x \succsim y$, then $v(x) \geq v(y)$ for every $v \in \mathcal{V}$ (because $\mathcal{V} \subseteq \mathcal{U}$ ). Conversely, if $v(x) \geq v(y)$ for every $v \in \mathcal{V}$, then denseness of $\mathcal{V}$ in $\mathcal{U}$ is easily seen to imply that $u(y)>u(x)$ cannot hold for any $u \in \mathcal{U}$, and hence, $x \succsim y$. Thus:

$$
x \succsim y \text { iff } v(x) \geq v(y) \text { for every } v \in \mathcal{V} .
$$

Let us now enumerate $\mathcal{V}$ as $\left\{v_{1}, v_{2}, \ldots\right\}$, and define $U:=\sum^{\infty} 2^{-i} v_{i}$. An immediate application of the Weierstrass $M$-Test shows that $U$ is a (well-defined) continuous [0,1]-valued map on $X$. Moreover, it is plain from (16) that $U$ is strictly $\succsim$-increasing.

Lemma B. Let $X$ be a locally compact and separable metric space, and $Y$ a nonempty compact subset of $X$. Let $\succsim$ be a continuous preorder on $X$. Then, there is a continuous and $\succsim$-increasing map $U: X \rightarrow[0,1]$ such that

$$
x \succ y \text { implies } U(x)>U(y)
$$

for every $x, y \in Y$.

Proof of Lemma B. Every locally compact and separable metric space is $\sigma$-compact, so there is a sequence $\left(K_{m}^{\prime}\right)$ of nonempty compact subsets of $X$ such that $K_{1}^{\prime} \subseteq K_{2}^{\prime} \subseteq \cdots$ and $X=\bigcup^{\infty} K_{i}^{\prime}$. Put $K_{i}:=K_{i}^{\prime} \cup Y$ for each $i$, and note that $Y \subseteq K_{1} \subseteq K_{2} \subseteq \cdots$ and $X=\bigcup^{\infty} K_{i}$. By Lemma A, there is a continuous $V: Y \rightarrow[0,1]$ such that $V(x)>V(y)$ whenever $x \succ y$ (for every $x, y \in Y)$. Let $\succsim_{i}:=\succsim \cap\left(K_{i} \times K_{i}\right)$ for each $i \in \mathbb{N}$, and note that, by the Nachbin Extension Theorem, there is a continuous and $\succsim_{1}$-increasing map $U_{1}: K_{1} \rightarrow[0,1]$ such that $\left.U_{1}\right|_{K_{1}}=V$. Similarly, there is a continuous and $\succsim_{2}$-increasing map $U_{2}: K_{2} \rightarrow[0,1]$ such that $\left.U_{2}\right|_{K_{2}}=U_{1}$, and so on. For any $x \in X$, we let $m(x)$ stand for the smallest integer such 
that $x \in K_{m(x)}$, and define $U: X \rightarrow[0,1]$ by $U(x):=U_{m(x)}$. Then, $U$ is a continuous and $\succsim$-increasing map that satisfies (17) for every $x, y \in Y$.

Proof of Levin's Theorem. We need to prove that there is a continuous and strictly $\succsim-$ increasing map $U: X \rightarrow[0,1]$. To this end, we again use $\sigma$-compactness of $X$ to find a sequence $\left(K_{m}\right)$ of nonempty compact subsets of $X$ such that $K_{1} \subseteq K_{2} \subseteq \cdots$ and $X=\bigcup^{\infty} K_{i}$. By Lemma $\mathrm{B}$, for every positive integer $i$, there is a continuous and $\succsim$-increasing map $U_{i}: X \rightarrow[0,1]$ such that

$$
x \succ y \text { implies } U_{i}(x)>U_{i}(y) \quad \text { for every } x, y \in K_{i} .
$$

Then, $U: X \rightarrow[0,1]$, defined by $U(x):=\sum^{\infty} 2^{-i} U_{i}$, is a continuous and strictly $\succsim$-increasing map.

We now turn to the proofs of Theorems 3 and 4 . We will actually prove Theorem 4 before Theorem 3, as it will be easier to deduce the latter from the former.

Proof of Theorem $4 .^{36}$ The "if" part of the assertion follows from Theorem 2. To prove its "only if" part, we apply Levin's Theorem to $\succsim^{*}:=\operatorname{tran}(R(c) \cup \unrhd)$ to find a continuous and strictly $\succsim^{*}$-increasing real map $u$ on $X$. As we have proved in the second paragraph of the proof of Theorem $2, \succsim^{*}$ is an extension of $\unrhd$. Therefore, $u$ is also strictly $\unrhd$-increasing. Finally, as $R(c) \subseteq \succsim^{*}$, we have $x \succsim^{*} y$, and hence, $u(x) \geq u(y)$, if there is an $A \in \mathcal{A}$ with $(x, y) \in c(A) \times A$. It follows that $c(A) \subseteq \arg \max \{u(x): x \in A\}$ for every $A \in \mathcal{A}$.

The following auxiliary lemma will be used in the proof of Theorem 3 .

Lemma C. Let $R, S$ and $T$ be binary relations on a metric space $X$ such that $R$ and $T$ are closed, and $S$ is compact, in $X \times X$. Then $R \circ S, S \circ R$ and $R \circ S \circ T$ are closed in $X \times X$.

Proof of Lemma C. We will only prove here that $R \circ S \circ T$ is closed; the arguments for the remaining cases are similar. Suppose $\left(x_{m}\right)$ and $\left(y_{m}\right)$ are two sequences that converge to $x$ and $y$, and we have $x_{m} R \circ S \circ T y_{m}$ for each $m$. Then, there are sequences $\left(z_{m}\right)$ and $\left(w_{m}\right)$ such that $x_{m} R z_{m} S w_{m} T y_{m}$. Since $S$ is compact, we may assume, after passing to subsequences if necessary, that $\left(z_{m}\right)$ and $\left(w_{m}\right)$ are convergent. Since $R, S$ and $T$ are closed in $X \times X$, we thus have $x R \lim z_{m} S \lim w_{m} T y$, which means $x R \circ S \circ T y$.

Proof of Theorem 3. The "if" part of the assertion follows from Theorem 2. To prove its "only if" part, we will use Lemma $\mathrm{C}$ to prove that $\operatorname{tran}(R(c) \cup \unrhd)$ is a continuous preorder on $X$. In view of Theorem 4, this will complete our proof.

We being by noting that $R(c)$ is a compact relation because $\mathcal{A}$ is a finite collection of compact sets and $c$ is compact-valued. Furthermore, $\unrhd$ is closed in $X \times X$ by assumption.

\footnotetext{
${ }^{36}$ While the compactness of the sets in $\mathcal{A}$ is part of the definition of a continuous choice environment, we do not rely on this property in this proof.
} 
Therefore, Lemma $\mathrm{C}$ guarantees that the relation $E^{1}:=\unrhd \circ R(c) \circ \unrhd$ is closed in $X \times X$. More generally,

$$
E^{k}:=\unrhd \circ R(c) \circ \unrhd \circ R(c) \circ \ldots \circ R(c) \circ \unrhd
$$

where $R(c)$ appears $k \in \mathbb{N}$ times on the right hand side is also closed relation in $X \times X$ (by repeated application of Lemma C). Suppose $x \operatorname{tran}(R(c) \cup \unrhd) y$. Then either $x \unrhd y$ or there is an integer $k \geq 4$ and $x_{1}, \ldots, x_{k} \in X$ such that

$$
x \unrhd x_{1} R(c) x_{2} \unrhd x_{3} R(c) \cdots R(c) x_{k} \unrhd y .
$$

In other words, $x E^{k} y$. Crucially, we can always ensure that $x E^{k} y$ for some $k \leq|\mathcal{A}|$. Indeed, if $x E^{k} y$ and $k>|\mathcal{A}|$, then there must be an $A \in \mathcal{A}$, and positive integers $i$ and $j$ such that $j>i$ and both $\left(x_{i}, x_{i+1}\right)$ and $\left(x_{j}, x_{j+1}\right)$ belong to $c(A) \times A$. In that case we can write

$$
x \unrhd x_{1} R(c) x_{2} \unrhd \cdots \unrhd x_{i} R(c) x_{j+1} \unrhd \cdots x_{k} \unrhd y,
$$

which means that $x E^{m} y$ for some $m \in\{1, \ldots, k-1\}$. We conclude that

$$
\operatorname{tran}(R(c) \cup \unrhd)=\unrhd \cup E^{1} \cup E^{2} \cup \cdots \cup E^{|\mathcal{A}|} .
$$

Therefore, $\operatorname{tran}(R(c) \cup \unrhd)$ is closed in $X \times X$, being the union of finitely many closed relations. In other words, $\operatorname{tran}(R(c) \cup \unrhd)$ is a continuous preorder on $X$, as we sought.

Proof of Proposition 5. Define $\succsim^{*}:=\operatorname{tran}(R(c) \cup \unrhd)$, and let $\succsim$ stand for the preference relation we have found in the second paragraph of the proof of Theorem 2. (If the conditions of Theorem 4 are satisfied, we let $\succsim$ be the preference relation that is induced by the map $u$ we found in the proof of Theorem 4.) We have seen in that paragraph that $\succsim$ extends $\unrhd$ and rationalizes $c$. Now take any $A \in \mathcal{A}$ and any $x \in A$. If $x \succsim^{*} y$ for some $y \in c(A)$, then $x \succsim y \succsim z$ for every $z \in A$, so $x \in \max (A, \succsim)$. Conversely, suppose $x \succsim^{*} y$ is false for any $y \in c(A)$, and pick an arbitrary $y$ in $c(A)$. Then, $y R(c) x$, so $y \succsim^{*} x$. It follows that $y \succ^{*} x$, and hence $y \succ x$, which means that $x$ does not belong to $\max (A, \succsim)$.

Proof of Proposition 6. Let $c$ be a choice correspondence on $\mathcal{A}$. Assume that $c$ satisfies the $\unrhd$-congruence axiom, and take any integer $k \geq 2$ and $A_{1}, \ldots, A_{k} \in \mathcal{A}$. Pick any $x_{1}, \ldots, x_{k} \in X$ with $x_{i} \in c\left(A_{i}\right) \times A_{i+1(\bmod k)}^{\downarrow}$ for each $i=1, \ldots, k$. Then, as the $\unrhd$-congruence axiom implies the cyclical $\unrhd$-consistency axiom (Proposition 1), (4) must hold. To prove (12), pick any $y \in A_{1}$ with $y \unrhd x_{k}$. As pointed out in the first paragraph of the proof of Proposition 1, we have $x_{k} \operatorname{tran}(R(c) \cup \unrhd) x_{1}$, and it follows that $y \operatorname{tran}(R(c) \cup \unrhd) x_{1}$. By the $\unrhd$-congruence axiom, therefore, $y \in c\left(A_{1}\right)$. Thus: $x_{k}^{\uparrow} \cap A_{1} \subseteq c\left(A_{1}\right)$. That $x_{i}^{\uparrow} \cap A_{i+1} \subseteq c\left(A_{i+1}\right)$ is analogously established for each $i=1, \ldots, k-1$.

Conversely, suppose that (3) implies (4) and (12), for every integer $k \geq 2$ and $A_{1}, \ldots, A_{k} \in \mathcal{A}$. Then, in particular, $c$ satisfies cyclical $\unrhd$-consistency. So, for any fixed, but arbitrary, $x, y \in X$ 
with $x \operatorname{tran}(R(c) \cup \unrhd) y$, Proposition 1 implies that $y \triangleright x$ cannot hold. To complete our proof, therefore, take any $A \in \mathcal{A}$, and assume that $x \in A$ and $y \in c(A)$. Given that $x \operatorname{tran}(R(c) \cup \unrhd)$ $y$, there exist an integer $k \geq 2$, and $x_{1}, \ldots, x_{k}$ and $y_{2}, \ldots, y_{k}$ in $X$, such that

$$
x \unrhd x_{k} R(c) y_{k} \unrhd x_{k-1} R(c) \cdots \unrhd x_{2} R(c) y_{2} \unrhd y=x_{1} .
$$

Then, for each $i=2, \ldots, k$, there is an $A_{i} \in \mathcal{A}$ such that $x_{i} \in c\left(A_{i}\right)$ and $y_{i} \in A_{i}$. In particular, $x_{1} \in A_{2}^{\downarrow}, x_{2} \in A_{3}^{\downarrow}, \ldots$, and $x_{k-1} \in A_{k}^{\downarrow}$. Setting $A_{1}:=A$, we also have $x_{k} \in A_{1}^{\downarrow}$. Thus, (3) holds, so, by hypothesis, (12) holds. In particular, $x_{k}^{\uparrow} \cap A_{1} \subseteq c\left(A_{1}\right)$, which implies that $x \in c(A)$, as we sought.

Proof of Theorem 7. The proof of the "if" part of the assertion is straightforward. To prove its "only if" part, we note that if $c$ satisfies the $\unrhd$-congruence axiom, then it is cyclically $\unrhd$-consistent, and hence there is a preference relation $\succsim$ on $X$ which extends $\unrhd$ and satisfies $c(A) \subseteq \max (A, \succsim)$ for every $A \in \mathcal{A}$ (Theorem 2). Moreover, as we have seen in Proposition 5, we can choose $\succsim$ such that (7) holds for every $A \in \mathcal{A}$ and $x \in A$. But then, for any $A \in \mathcal{A}$, if $x \in \max (A, \succsim)$, then $x \operatorname{tran}(R(c) \cup \unrhd) y$ for some $y \in c(A)$, and hence, $x \in c(A)$ by the $\unrhd$-congruence axiom. Thus, $c(A)=\max (A, \succsim)$ for every $A \in \mathcal{A}$, and we are done.

Proof of Theorem 8. We only need to prove the "only if" part of the assertion. For this, it is enough to apply the argument given in the proof of Theorem 7 (but this time use the second part of Proposition 5 as well) to find a continuous utility function that strictly $\unrhd$-rationalizes $c$.

Proof of Proposition 10. (i) Suppose $\mathbf{x}$ is not revealed preferred to $\mathbf{y}$. In that case, put $\mathcal{A}^{*}:=\mathcal{A} \cup\{\mathbf{x}, \mathbf{y}\}$, where $\mathcal{A}=\{B(\mathbf{p}, 1): \mathbf{p} \in P\}$, and define the correspondence $c^{*}: \mathcal{A}^{*} \rightrightarrows \mathbb{R}_{+}^{n}$ by $\left.c^{*}(B(\mathbf{p}, 1))\right):=d(\mathbf{p})$ for each $p \in P$, and $c^{*}(\{\mathbf{x}, \mathbf{y}\}):=\mathbf{y}$. Since $\mathbf{x}$ is not revealed preferred to $\mathbf{y}$, that is, $(\mathbf{x}, \mathbf{y})$ is not in $\operatorname{tran}(R(c) \cup \geq)$, and $c$ obeys cyclical $\geq$-consistency, $c^{*}$ also obeys cyclical $\geq$-consistency. By construction, $(\mathbf{y}, \mathbf{x}) \in R\left(c^{*}\right)$ but $(\mathbf{x}, \mathbf{y})$ does not belong to $\operatorname{tran}\left(R\left(c^{*}\right) \cup \geq\right)$. By Proposition 5 there exists a continuous function $u^{*}$ that $\geq$-rationalizes $c^{*}$, and hence $u^{*} \in \mathcal{U}(d)$, such that $u^{*}(\mathbf{y})>u^{*}(\mathbf{x})$. We conclude that $(\mathbf{x}, \mathbf{y})$ does not belong to $S(d)$.

(ii) We define $\mathcal{A}^{*}$ and $c^{*}$ as in the proof of (i). If $\mathbf{x}$ is not revealed preferred to $\mathbf{y}$, then we know from the proof of (i) that $(\mathbf{x}, \mathbf{y})$ is not in $S(d)$ and hence not in $S^{\prime}(d)$. Now suppose $\mathbf{x}$ is revealed preferred to $\mathbf{y}$, but not strictly so. In that case, one could check that $c^{*}$ still satisfies cyclical $\geq$-consistency and hence Theorem 3 tells us that there is a continuous function $u^{* *}$ that $\geq$-rationalizes $c^{*}$. Since $\mathbf{x} \operatorname{tran}\left(R\left(c^{*}\right) \cup \geq\right) \mathbf{y}$, and by construction, $\mathbf{y} R\left(c^{*}\right) \mathbf{x}$, we obtain $u^{* *}(\mathbf{x})=u^{* *}(\mathbf{y})$. As $u^{* *} \in \mathcal{U}(d)$, it follows that $(\mathbf{x}, \mathbf{y})$ does not belong to $S^{\prime}(d) .{ }^{37}$

\footnotetext{
37 An alternative way of proving this result is to first notice that the set of preferences that $\geq$ rationalize $d$ are precisely those preferences that extend $\operatorname{tran}(R(c) \cup \geq$ ) (see the proof of Theorem 2).
} 
Proof of Proposition 11. The "only if" parts of the claims in both (i) and (ii) are straightforward. By the definition of $d, \mathbf{p x}=I$ for all $\mathbf{x} \in d(\mathbf{p}, I)$; as we have explained in Section 4.4 , this property of $d$, together with the congruence axiom, guarantees that $d$ obeys the $\geq$-congruence axiom. Therefore, by Theorem 4, the 'if' part of the claims in both (i) and (ii) hold if $\operatorname{tran}(R(c) \cup \geq)$ is continuous for the choice correspondence $c: B(\mathbf{p}, I) \mapsto d(\mathbf{p}, I)$ on $\mathcal{A}$. Note that, if $\mathbf{y} \operatorname{tran}(R(c) \cup \geq) \mathbf{z}$, then there are $\left(\mathbf{p}^{1}, I_{1}\right), \ldots,\left(\mathbf{p}^{k}, I_{k}\right) \in P \times \mathbb{I}$ and $\mathbf{x}^{1} \in d\left(\mathbf{p}^{1}, I_{1}\right), \ldots, \mathbf{x}^{k} \in d\left(\mathbf{p}^{k}, I_{k}\right)$ such that

$$
\mathbf{y} \geq \mathbf{x}^{1}, \mathbf{p}^{1} \mathbf{x}^{1} \geq \mathbf{p}^{1} \mathbf{x}^{2}, \ldots, \mathbf{p}^{k-1} \mathbf{x}^{k-1} \geq \mathbf{p}^{k-1} \mathbf{x}^{k} \text { and } \mathbf{p}^{k} \mathbf{x}^{k} \geq \mathbf{p}^{k} \mathbf{z}
$$

Crucially, we may choose $\mathbf{p}^{1}, \ldots, \mathbf{p}^{k}$ to be distinct. Indeed, cyclical $\geq$-consistency requires that if $\mathbf{p}^{r}=\mathbf{p}^{s}$ for some $r<s$, then $I_{r} \geq I_{s}$; thus $B\left(\mathbf{p}^{s}, I_{s}\right) \subseteq B\left(\mathbf{p}^{r}, I_{r}\right)$ and it follows that $\mathbf{p}^{r} \mathbf{x}^{r} \geq \mathbf{p}^{r} \mathbf{x}^{s+1}$, that is, we may 'snip off' the part of the sequence in (18) between $r+1$ and $s$.

Take any $\mathbf{y}, \mathbf{y}_{1}, \mathbf{y}_{2}, \ldots, \mathbf{z}, \mathbf{z}_{1}, \mathbf{z}_{2}, \ldots \in \mathbb{R}_{+}^{n}$ such that $\mathbf{y}_{m} \rightarrow \mathbf{y}, \mathbf{z}_{m} \rightarrow \mathbf{z}$ and $\mathbf{y}_{m} \operatorname{tran}(R(c) \cup$ $\geq) \mathbf{z}_{m}$ for each $m$. We wish to show that $\mathbf{y} \operatorname{tran}(R(c) \cup \geq) \mathbf{z}$. Indeed, each pair of $\mathbf{y}_{m}$ and $\mathbf{z}_{m}$ is linked by a sequence of inequalities like (18), where the price vectors in that sequence are distinct. This property, together with the finiteness of $P$, guarantees that we can find subsequences $\mathbf{y}_{m_{t}}$ and $\mathbf{z}_{m_{t}}$ and distinct vectors $\mathbf{p}^{1}, \ldots, \mathbf{p}^{k}$ in $P$ such that

$$
\mathbf{y}_{m_{t}} \geq \mathbf{x}_{m_{t}}^{1}, \mathbf{p}^{1} \mathbf{x}_{m_{t}}^{1} \geq \mathbf{p}^{1} \mathbf{x}_{m_{t}}^{2}, \ldots, \mathbf{p}^{k-1} \mathbf{x}_{m_{t}}^{k-1} \geq \mathbf{p}^{k-1} \mathbf{x}_{m_{t}}^{k} \text { and } \mathbf{p}^{k} \mathbf{x}_{m_{t}}^{k} \geq \mathbf{p}^{k} \mathbf{z}_{m_{t}}
$$

By the upper hemicontinuity of $d$, and after passing to further subsequences if necessary, we obtain $I_{l, m_{t}} \rightarrow I_{l}>0$ and $\mathbf{x}_{m_{t}}^{l} \rightarrow \mathbf{x}^{l} \in d\left(\mathbf{p}^{l}, I_{l}\right)$. Thus,

$$
\mathbf{y} \geq \mathbf{x}^{1}, \mathbf{p}^{1} \mathbf{x}^{1} \geq \mathbf{p}^{1} \overline{\mathbf{x}}^{2}, \ldots, \mathbf{p}^{k-1} \mathbf{x}^{k-1} \geq \mathbf{p}^{k-1} \mathbf{x}^{k} \text { and } \mathbf{p}^{k} \mathbf{x}^{k} \geq \mathbf{p}^{k} \mathbf{z}
$$

which means that $\mathbf{y} \operatorname{tran}(R(c) \cup \geq) \mathbf{z}$, as we sought.

\section{REFERENCES}

Andreoni, J. and C. Sprenger, Estimating time preferences from convex budgets, American Economic Review, 102 (2012), 3333-3356.

Afriat, S., The construction of a utility function from demand data, International Economic Review, 8 (1967), 67-77.

Arrow, K., Rational choice functions and orderings, Economica 26 (1959), 121-27.

One could then appeal to a result in Evren and Ok (2011, Corollary 1), which says that any closed preorder in a locally compact and separable metric space is the intersection of all the preferences representable by continuous utility functions that extend the preorder. Applying this result to $\operatorname{tran}(R(c) \cup \geq)$ gives us the conclusion we want. Note that it is also known that any preorder defined on an arbitrary set is the intersection of all preferences that extend it (see Donaldson and Weymark (1998) and Dushnik and Miller (1941)). Therefore, even if assumptions are not made to guarantee that $\operatorname{tran}(R(c) \cup \geq)$ is closed, we would still know that it is the intersection of all preferences that extend it. 
Blundell, R. W., M. Browning and I. A. Crawford, Nonparametric Engel curves and revealed preference, Econometrica, 71 (2003), 205-40.

Bossert, W.and K. Suzumura, Revealed preference and choice under uncertainty, SERIEs, 3 (2012), 247-258.

Bridges, D. and G. Mehta, Representations of Preference Orderings, Lecture Notes in Economics and Mathematical Systems, Vol. 422, Springer, Berlin, 1996.

Browning, M., A nonparametric test of the life-cycle rational expectations hypothesis, International Economic Review, 30 (1989), 979-992.

Chavas, J.-P. and T. Cox, On generalized revealed preference analysis, Quarterly Journal of Economics, 108 (1993), 493-506.

Choi, S., R. Fisman, D. Gale, and S. Kariv, Consistency and heterogeneity of individual behavior under uncertainty, American Economic Review, 97 (2007), 1921-1938.

Demuynck, T., Absolute and relative time-consistent revealed preferences, Theory and Decision, 66 (2009), 283-299.

Donaldson, D. and J. A. Weymark, A quasi-ordering is the intersection of orderings, Journal of Economic Theory, 78 (1988), 382-387.

Dushnik, B. and E. W. Miller, Partially ordered sets, American Journal of Mathematics, 63 (1941) 600-610.

Dziewulski, P., Revealed time preference, Oxford Department of Economics Discussion Papers, 748 (2015).

Echenique, F., T. Imai and K. Saito, Testable implications of models of intertemporal choice: Exponential discounting and its generalizations, Caltech SS Working Papers, 1388 (2015).

Evren, O. and E.A. Ok, On the multi-utility representation of preference relations, Journal of Mathematical Economics, 47 (2011), 554-63.

Fishburn, P.C., Separation theorems and expected utilities, Journal of Economic Theory, 11 (1975), 16-34.

Forges, F. and Minelli, E., Afriat's theorem for general budget sets, Journal of Economic Theory, 144 (2009), 135-45.

Frederick, S., G. Loewenstein, and T. O'Donoghue, Time discounting and time preference: A critical review, Journal of Economic Literature, 40 (2002), 351-401.

Green, E. J. and K. Osband, A revealed preference theory for expected utility, Review of Economic Studies, 58 (1991), 677-695.

Green, R. C., and S. Srivastava, Expected utility maximization and demand behavior, Journal of Economic Theory, 38 (1986), 313-23.

Halevy, Y., D. Persitz, and L Zrill, Parametric recoverability of preferences, Working Paper, Vancouver School of Economics (2014).

Herden, G., On the existence of utility functions, Mathematical Social Sciences, 17 (1989), 
297-313.

Herden, G. and A. Pallack, On the continuous analogue of the Sprilrajn Theorem I. Mathematical Social Sciences, 43 (2002), 115-134.

Heufer, J., Nonparametric comparative revealed risk aversion, Journal of Economic Theory, 153, (2014), 569-616.

Houthakker, H., Revealed preference and the utility function, Economica, 17 (1950), 159 -174.

Kalandrakis, T., Rationalizable voting, Theoretical Economics, 5 (2010), 93-125.

Levin, V., Measurable utility theorems for closed and lexicographic preference relations, Soviet Mathematics Doklady, 27 (1983), 639-643.

Mas-Colell, A., On revealed preference analysis, Review of Economic Studies, 45 (1978), 121131.

Matzkin, R., Axioms of revealed preferences for nonlinear choice sets, Econometrica, 59 (1991), 1779-86.

Nash, J., The bargaining problem, Econometrica, 18 (1950), 155-62.

Reny, P., A characterization of rationalizable consumer behavior, Econometrica, 83 (2015). $175-92$.

Richter, M., Revealed preference theory, Econometrica, 34 (1966), 635-45.

Samuelson, P., A note on the pure theory of consumer's behavior, Economica, 5 (1938), 61-71.

Stiglitz, J., On the irrelevance of corporate financial policy, American Economic Review, 64 (1974), 851-66.

Varian, H., The nonparametric approach to demand analysis, Econometrica, 50 (1982), 945-73.

Varian, H., Non-parametric tests of consumer behaviour, Review of Economic Studies, 50 (1983), 99-110. 